\title{
Combination of Antiviral Drugs to Inhibit SARS-CoV-2 Polymerase and Exonuclease as Potential COVID-19 Therapeutics
}

Xuanting Wang ${ }^{1,2, a}$, Carolina Q. Sacramento ${ }^{3,4, a}$, Steffen Jockusch ${ }^{1,5, a}$, Otávio Augusto Chaves ${ }^{3,4}$, Chuanjuan Tao ${ }^{1,2}$, Natalia Fintelman-Rodrigues ${ }^{3,4}$, Minchen Chien ${ }^{1,2}$, Jairo R. Temerozo ${ }^{6,7}$, Xiaoxu $\mathrm{Li}^{1,2}$, Shiv Kumar ${ }^{1,2}$, Wei Xie ${ }^{9}$, Dinshaw J. Patel ${ }^{9}$, Cindy Meyer ${ }^{10}$, Aitor Garzia $^{10}$, Thomas Tuschl ${ }^{10}$, Patrícia T. Bozza ${ }^{3}$, James J. Russo ${ }^{1,2}$, Thiago Moreno L. Souza ${ }^{3,4, b_{*}}$, Jingyue $\mathrm{Ju}^{1,2,8, \mathrm{~b}_{*}}$

1 - Center for Genome Technology and Biomolecular Engineering, Columbia University, New York, NY 10027.

2 - Department of Chemical Engineering, Columbia University, New York, NY 10027.

3 - Laboratory of Immunopharmacology, Oswaldo Cruz Institute (IOC), Oswaldo Cruz Foundation (Fiocruz), Rio de Janeiro, RJ, Brazil.

4 - National Institute for Science and Technology for Innovation on Neglected Diseases (INCT/IDN), Center for Technological Development in Health (CDTS), Oswaldo Cruz Foundation (Fiocruz), Rio de Janeiro, RJ, Brazil.

5 - Department of Chemistry, Columbia University, New York, NY 10027.

6 - Laboratory on Thymus Research, Oswaldo Cruz Institute (IOC), Oswaldo Cruz Foundation (Fiocruz), Rio de Janeiro, RJ, Brazil.

7 - National Institute for Science and Technology on Neuroimmunomodulation (INCT/NIM), Oswaldo Cruz Institute (IOC), Oswaldo Cruz Foundation (Fiocruz), Rio de Janeiro, RJ, Brazil.

8 - Department of Molecular Pharmacology and Therapeutics, Columbia University, New York, NY 10032.

9 - Laboratory of Structural Biology, Memorial Sloan-Kettering Cancer Center, New York, NY 10065.

10 - Laboratory of RNA Molecular Biology, Rockefeller University, New York, NY 10065.

a - These authors contributed equally

b - These senior authors contributed equally

*To whom correspondence should be addressed. Email: dj222@columbia.edu (JJ); tmoreno@cdts.fiocruz.br (TMLS) 


\begin{abstract}
SARS-CoV-2 has an exonuclease-based proofreader, which removes nucleotide inhibitors such as Remdesivir that are incorporated into the viral RNA during replication, reducing the efficacy of these drugs for treating COVID-19. Combinations of inhibitors of both the viral RNA-dependent RNA polymerase and the exonuclease could overcome this deficiency. Here we report the identification of hepatitis $\mathrm{C}$ virus NS5A inhibitors Pibrentasvir and Ombitasvir as SARS-CoV-2 exonuclease inhibitors. In the presence of Pibrentasvir, RNAs terminated with the active forms of the prodrugs Sofosbuvir, Remdesivir, Favipiravir, Molnupiravir and AT-527 were largely protected from excision by the exonuclease, while in the absence of Pibrentasvir, there was rapid excision. Due to its unique structure, Tenofovir-terminated RNA was highly resistant to exonuclease excision even in the absence of Pibrentasvir. Viral cell culture studies also demonstrate significant synergy using this combination strategy. This study supports the use of combination drugs that inhibit both the SARS-CoV-2 polymerase and exonuclease for effective COVID-19 treatment.
\end{abstract}

\title{
Introduction
}

Severe acute respiratory syndrome coronavirus 2 (SARS-CoV-2), which is responsible for COVID19 , is a positive-sense single-stranded RNA virus ${ }^{1,2}$. Thus it requires an RNA-dependent RNA polymerase $(\mathrm{RdRp})$ to replicate and transcribe its genome. Because of its large genome $(\sim 30 \mathrm{~kb})$ and error-prone RdRp, SARS-CoV-2 also possesses a 3'-5' exonuclease for proofreading to maintain the integrity of the genome ${ }^{3}$. The replication complex of coronaviruses consists of several viral proteins, including the RdRp itself (nonstructural protein 12; nsp12) and its two accessory proteins (nsp7 and nsp8) $)^{4-6}$, and the exonuclease (nsp14) with its accessory protein (nsp10) ${ }^{7}$.

While a variety of drugs targeting many of the SARS-CoV-2 proteins essential for its infectious cycle have been evaluated, to date no effective antiviral strategy for COVID-19 exists. Remdesivir (RDV), a nucleotide analogue, is the only small molecule antiviral drug approved by the Food and Drug Administration (FDA) for COVID-19 treatment, although it was shown to have limited benefit in the World Health Organization's Solidarity Trial. Lack of oral bioavailability and short half-life reduces RDV's clinical effectiveness. With the appearance of new SARS-CoV-2 variants that pose a threat to public health and may escape the immune response to current vaccines, it is more critical than ever to develop antivirals that can treat COVID-19 effectively. We have investigated a library of nucleotide analogues targeting the SARS-CoV-2 RdRp, several of which have been FDA approved for other viral infections including HIV/AIDS and hepatitis B and $\mathrm{C}^{8-11}$. Of particular interest are those that are able to be incorporated into the replicated RNA by the viral RdRp where they halt or slow further replication. These include, among several others, nucleotide analogues such as Sofosbuvir (Fig. 1a), an immediate terminator of the polymerase reaction which has since entered COVID-19 clinical trials ${ }^{12,13}$, Remdesivir (Fig. 1b), a delayed terminator ${ }^{14,15}$, and Tenofovir, an obligate terminator (Fig. 1d) ${ }^{10,11}$. Recently, we demonstrated that upon incorporation of the triphosphate forms of Sofosbuvir and Remdesivir into RNA by the SARS-CoV-2 RdRp complex, both nucleotide analogues were removed by the SARS-CoV-2 exonuclease complex, although Sofosbuvir was removed at a slower rate ${ }^{16}$. Currently, three other prodrugs related to nucleotides, Favipiravir (Fig. 1c), Molnupiravir and AT-527 (Fig. 1e, f), are undergoing clinical trials for COVID-19. 


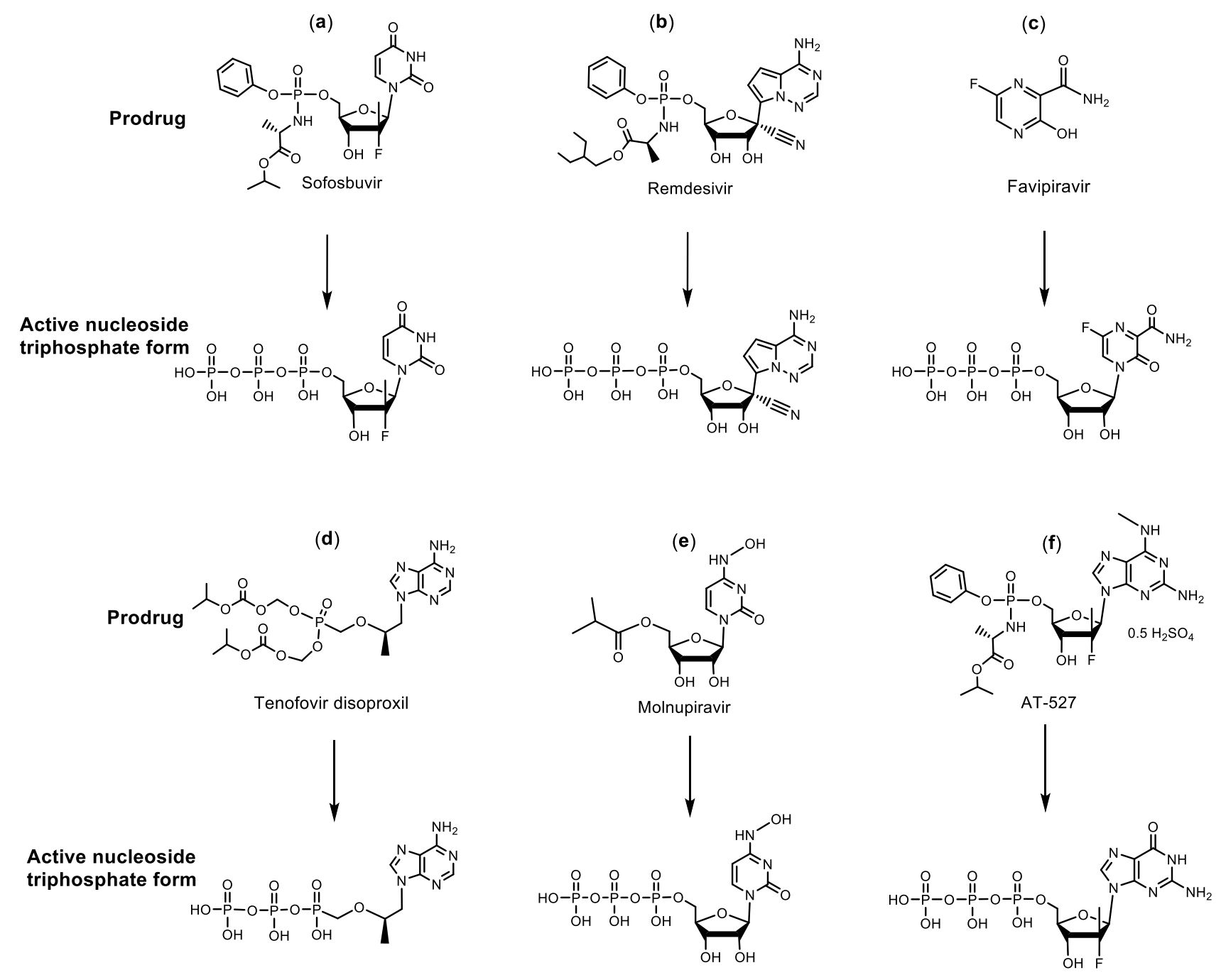

Fig. 1 Structures of the prodrugs Sofosbuvir (a), Remdesivir (b), Favipiravir (c), Tenofovir disoproxil (d), Molnupiravir (e) and AT-527 (f) (top) and their respective active triphosphate forms Sofosbuvir-5'-triphosphate, Remdesivir-5'-triphosphate, Favipiravir-ribofuranosyl-5'triphosphate (Favipiravir-RTP), Tenofovir diphosphate, $\mathbf{N}^{4}$-hydroxycytidine-5'-triphosphate (NHC-TP) and 2'-fluoro-2'-methyl guanosine-5'-triphosphate (AT-9010, $\mathbf{G}_{\mathrm{fm}}$-TP) (bottom).

While nucleoside/nucleotide analogues show promising results against SARS-CoV-2, in vitro pharmacological parameters often exceed human plasma exposure. Most likely, nucleoside/nucleotide-based inhibitors are insufficient to block viral RNA replication, because they could be quickly excised by the SARS-CoV-2 exonuclease. Combination of RdRp inhibitors such as Sofosbuvir, Remdesivir and others listed in Fig. 1 with exonuclease inhibitors may provide a more effective strategy for blocking SARS-CoV-2 RNA replication. We previously described the need to overcome the exonuclease proofreading function in order to completely inhibit SARS-CoV-2 replication in early 2020. ${ }^{10}$ An in silico study has proposed combining RdRp and exonuclease inhibitors as a strategy to combat COVID-19 ${ }^{17}$; however, no successful demonstration of such a combined drug strategy has been reported. Hepatitis $\mathrm{C}$ virus (HCV) and SARS-CoV-2 are both positive sense single stranded RNA viruses. Viruses with small genomes that encode relatively few 
proteins, such as $\mathrm{HCV}$, must possess multifunctional proteins ${ }^{18}$. $\mathrm{HCV}$ NS5A is a good example because it binds lipids, $\mathrm{Zn}^{++}$and RNA, as well as participates in phosphorylation and cell signaling events $^{18}$. The SARS-CoV-2 genome is 3-fold larger than that of HCV, and the analogous NS5A functions are likely distributed among proteins nsp1 to nsp16 of the coronavirus ${ }^{19}$. We have previously shown that the NS5A inhibitors Daclatasvir and Velpatasvir inhibit both the SARS-CoV2 polymerase and exonuclease activities ${ }^{20,21}$. The combination of Velpatasvir with Remdesivir was shown to have a synergistic effect relative to Remdesivir alone in inhibiting SARS-CoV-2 replication $^{22}$. Here we further examined whether the NS5A inhibitors Pibrentasvir, Ombitasvir and Daclatasvir (structures shown in Fig. S-1), possess anti-exonuclease activity and could synergize with RdRp inhibitors in virus replication assays. We discovered that Pibrentasvir and Ombitasvir have the highest exonuclease inhibitory activity among all the NS5A inhibitors we have tested. As an example, we show here that Pibrentasvir reduced the removal by exonuclease of immediate and delayed RNA chain terminators incorporated into the RNA. Consequently, Pibrentasvir and Ombitasvir synergistically improved the in vitro pharmacological parameters of several RdRp inhibitors, such as Sofosbuvir, Remdesivir, Tenofovir and Favipiravir. Since these RdRp inhibitors are clinically approved antivirals, candidate combinations of the polymerase and exonuclease inhibitors identified here could be prioritized for advancement to COVID-19 clinical trials.

\section{Results}

\section{Molecular docking study of HCV NS5A inhibitors with SARS-CoV-2 exonuclease nsp14.}

We performed a molecular docking study to investigate whether the HCV NS5A inhibitors (Fig. S1) interact with the SARS-CoV-2 exonuclease. Since a 3D structure for SARS-CoV-2 nsp14 was not yet available, we built a protein model based on the ortholog in SARS-CoV ${ }^{3}$. Superposition of the $3 \mathrm{D}$ structures of these two enzymes indicates that the main domains and the catalytic amino acid residues of the exonuclease active sites are structurally similar (Fig. S-2). The clinically approved HCV NS5A inhibitors are bound to the SARS-CoV-2 nsp14 exonuclease active site with comparable docking scores (Fig. 2). The exonuclease activity requires the proper coordination of the $\mathrm{Mg}^{++}$ion with amino acid residues Asp-90, Glu-92, Glu-191 and Asp-273 as well as the RNA substrate. NS5A inhibitors interfere with the coordination among the $\mathrm{Mg}^{++}$ion, the amino acids in the nsp14 active site and the RNA 3' terminus, likely preventing nucleotide excision from the RNA (Fig. 2).

\section{Inhibition of SARS-CoV-2 exonuclease activity by HCV NS5A inhibitors to prevent the excision of immediate and delayed terminators from RNA.}

Using the molecular assays that we have developed previously ${ }^{16}$, we evaluated if HCV NS5A inhibitors could indeed inhibit SARS-CoV-2 exonuclease activity. We identified Pibrentasvir and Ombitasvir as two strong inhibitors of the SARS-CoV-2 exonuclease. As a proof of principle, we used Pibrentasvir to demonstrate whether such drugs can prevent excision of nucleotide analogues from RNAs terminated with the active forms of prodrugs such as Remdesivir, Sofosbuvir, Tenofovir, Favipiravir, Molnupiravir and AT-527. 


Docking score (-KJ/mol)
according to docking space
\begin{tabular}{|c|c|c|}
\hline Antiviral & $8 \AA$ & $10 \AA$ \\
\hline Ombitasvir & 20.36 & 62.64 \\
\hline Elbasvir & 55.38 & 60.83 \\
\hline Ledipasvir & 12.14 & 57.78 \\
\hdashline Velpatasvir & 16.33 & 57.29 \\
\hdashline Daclatasvir & 18.41 & 49.25 \\
\hline Pibrentasvir & 17.89 & 47.12 \\
\hline
\end{tabular}

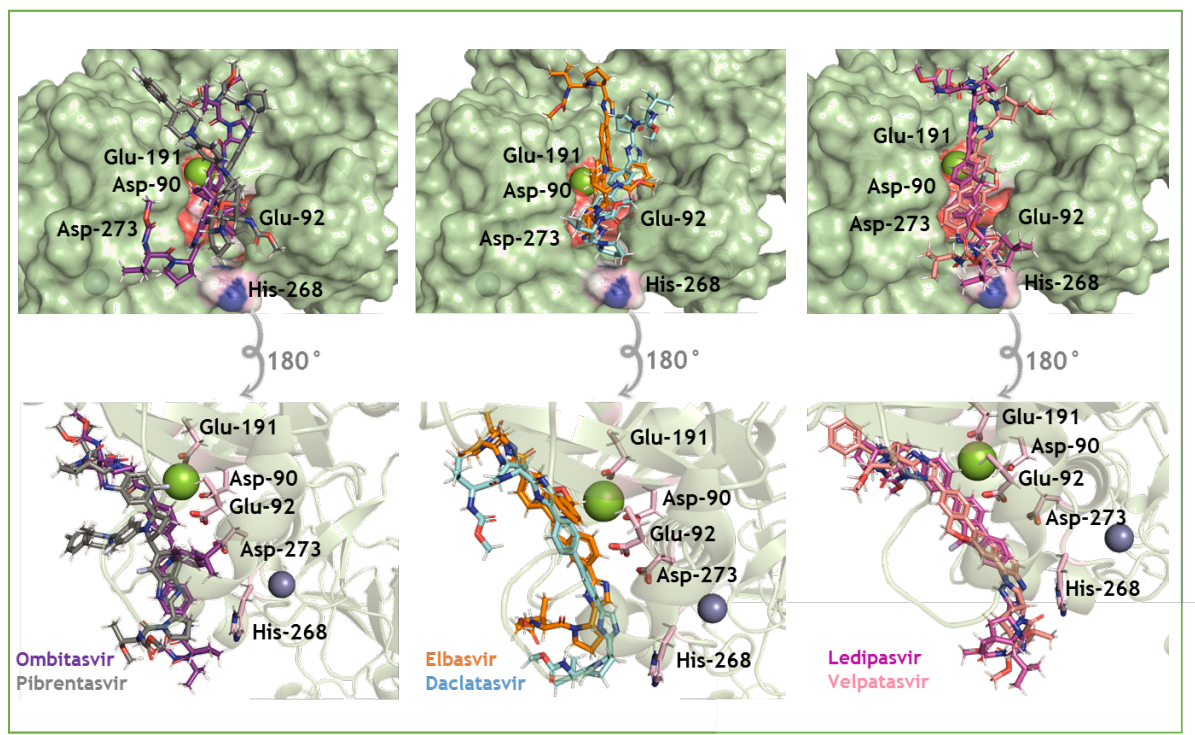

Fig. 2 3D representation of the best docking poses for HCV NS5A inhibitors in the nsp14 exonuclease active site. The NS5A inhibitors were built and minimized in terms of energy by Density Functional Theory (DFT). Docking was performed using GOLD 2020.2 software with ChemPLP as scoring function. Because of the diversity in NS5A inhibitors' molecular weights, they were allowed to dock within 8 or $10 \AA$ spheres in the SARS-CoV-2 nsp14 exonuclease active site, constructed in Fig. S-2. The NS5A and catalytic amino acid residues are in stick representation. $\mathrm{Mg}^{++}$and $\mathrm{Zn}^{++}$are represented as green and indigo blue spheres, respectively.

Pibrentasvir and Ombitasvir inhibit the SARS-CoV-2 exonuclease in a concentration dependent manner, as shown in Fig. 3. RNA (sequence shown at the top of the figure) was incubated with a SARS-CoV-2 pre-assembled exonuclease complex (nsp14/nsp10) at $37^{\circ} \mathrm{C}$ for $15 \mathrm{~min}$ in the absence (b) and presence of varying amounts of Pibrentasvir (c-e). The RNA (a) and the products of the exonuclease reaction (b-e) were analyzed by MALDI-TOF MS. The peak at 8160 Da corresponds to the intact RNA. In the absence of Pibrentasvir, exonuclease activity caused nucleotide cleavage from the 3'-end of the RNA as shown by the 7 lower molecular weight fragments corresponding to cleavage of 1-7 nucleotides (b), with only $\sim 7 \%$ intact RNA remaining (peak at 8167 Da). Pibrentasvir $(1,10$ and $20 \mu \mathrm{M})$ reduced exonuclease activity in a concentration dependent manner as shown by the reduced intensities of the fragmentation peaks and more prominent intact RNA peak (c-e). Ombitasvir displayed similar efficiency of exonuclease activity inhibition (Fig. 3 f-i) as Pibrentasvir. Daclatasvir also exhibits inhibition of exonuclease activity, but at higher concentrations than either Pibrentasvir or Ombitasvir (Fig. S-3). 

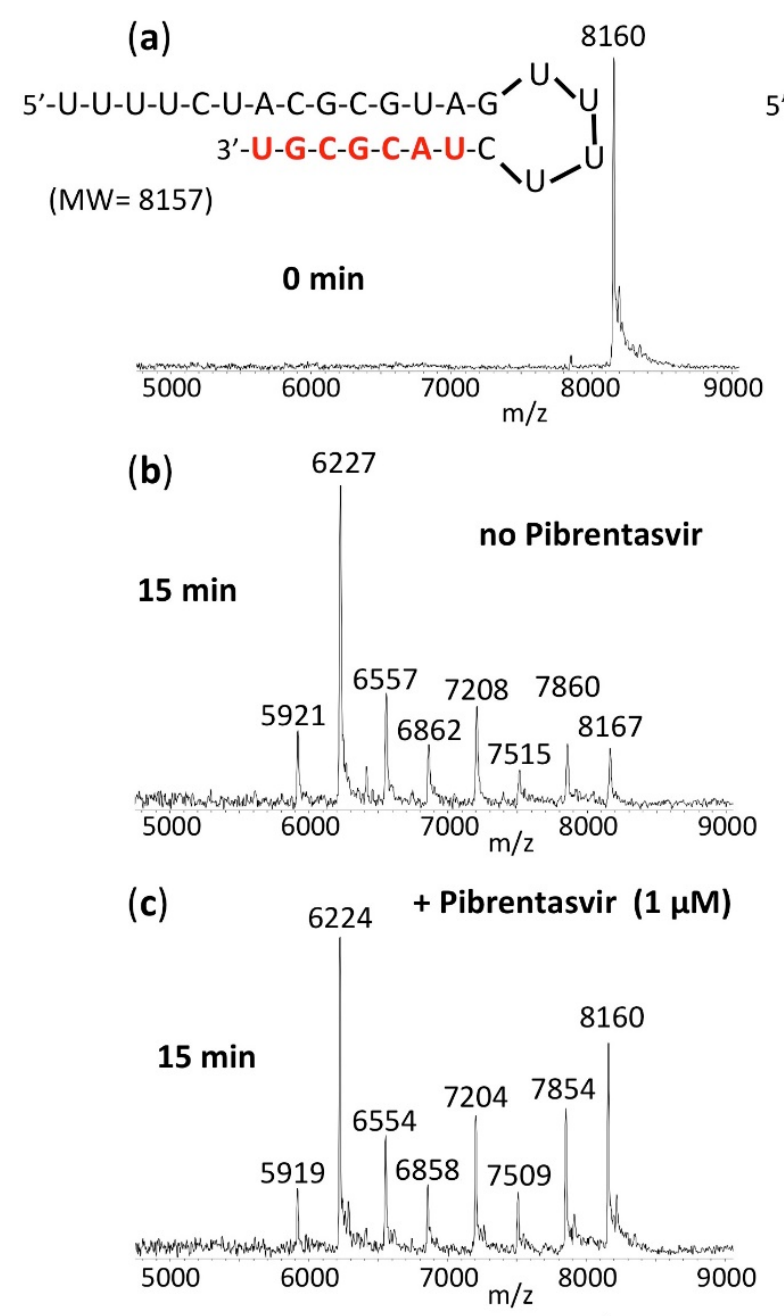

(d) + Pibrentasvir $(\mathbf{1 0} \mu \mathrm{M}) \quad 8165$
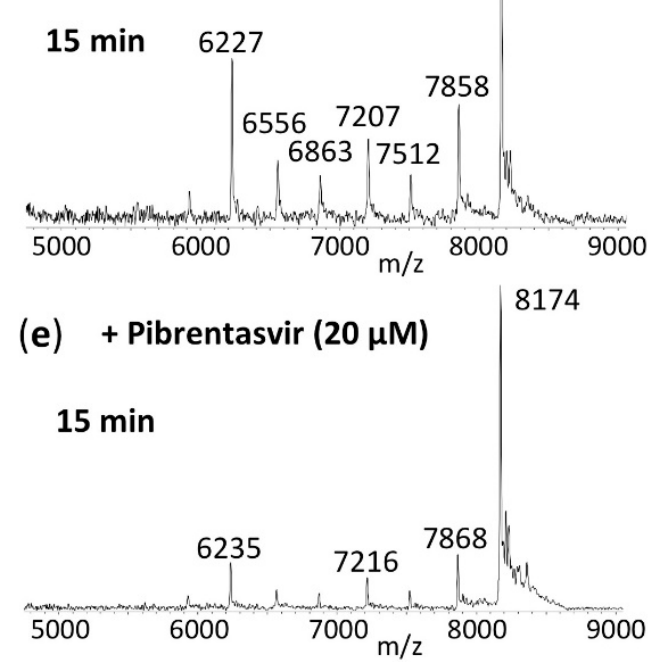

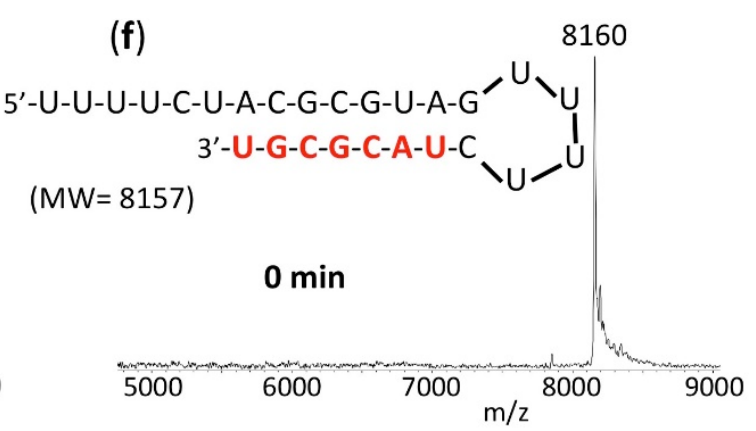

(g)

no Ombitasvir

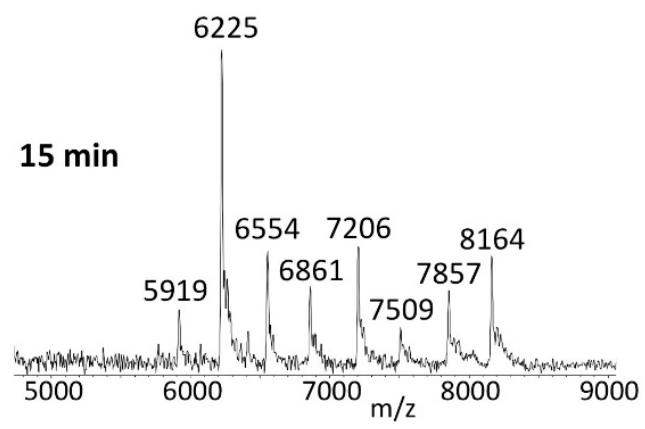

(h) + Ombitasvir (10 $\mu \mathrm{M})$

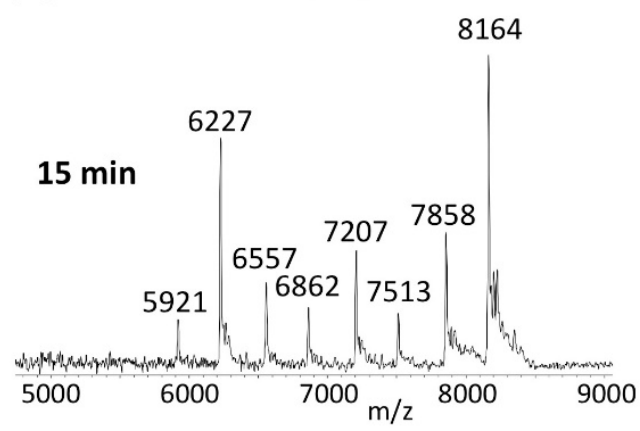

(i) + Ombitasvir $(20 \mu \mathrm{M})$

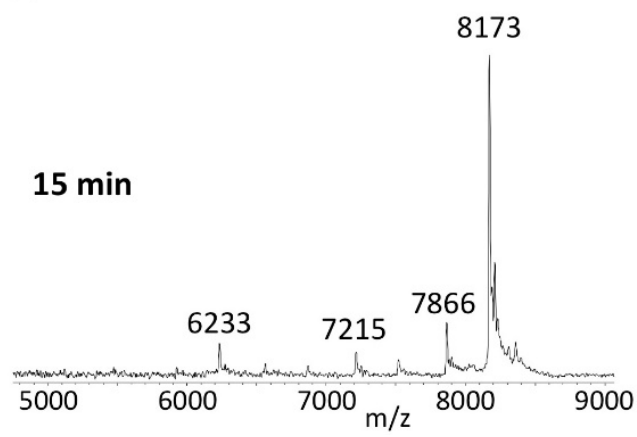

Fig. 3 Inhibition of SARS-CoV-2 exonuclease activity by Pibrentasvir and Ombitasvir. A mixture of $500 \mathrm{nM}$ RNA (sequence shown at the top of the figure) and $50 \mathrm{nM}$ SARS-CoV-2 preassembled exonuclease complex (nsp14/nsp10) was incubated in buffer solution at $37^{\circ} \mathrm{C}$ for 15 min 
in the absence $(b, g)$ and presence of varying amounts of Pibrentasvir (c-e) and Ombitasvir (h, i). The RNA $(a, f)$ and the products of the exonuclease reaction (b-e, g-i) were analyzed by MALDITOF MS. The signal intensity was normalized to the highest peak. The peak at 8160 Da corresponds to the intact RNA (8157 Da expected). In the absence of Pibrentasvir, exonuclease activity caused nucleotide cleavage from the 3'-end of the RNA as shown by the 7 lower molecular weight fragments corresponding to cleavage of 1-7 nucleotides (b). Under our experimental conditions, approximately 7\% intact RNA remained as indicated by the peak at $8167 \mathrm{Da}$ (b). With increasing amounts of Pibrentasvir, exonuclease activity was reduced as shown by the reduced intensities of the fragmentation peaks and increased intact RNA peak (c-e). Similar results were observed with Ombitasvir (f-i).

We next determined if RNA terminated with the active forms of prodrugs such as Sofosbuvir, Remdesivir, Tenofovir, Favipiravir, Molnupiravir and AT-527 were also less likely to be excised by SARS-CoV-2 exonuclease in the presence of Pibrentasvir. We generated Sofosbuvir and Remdesivir terminated RNA by single base extension of RNA template-loop-primers with the active triphosphate forms of Sofosbuvir and Remdesivir, respectively, using the replication complex assembled from SARS-CoV-2 nsp12 (the viral RdRp) and nsp7 and nsp8 proteins (RdRp cofactors) ${ }^{11,16}$. The Sofosbuvir (S)-terminated RNA and Remdesivir (R)-terminated RNA (sequences shown at the top of Fig. 4) were incubated with the SARS-CoV-2 nsp14/nsp10 complex at $37{ }^{\circ} \mathrm{C}$ for $15 \mathrm{~min}$ in the absence (Fig. 4b, e) and presence of $20 \mu \mathrm{M}$ Pibrentasvir (c, f). MALDI-TOF MS analysis of the intact RNAs (a, d) and the products of the exonuclease reactions (b, c, e, f) was performed. In the absence of Pibrentasvir, exonuclease activity caused cleavage of 1-7 nucleotides from the 3'-end of the RNA as shown by the lower molecular weight fragments (b, e). When $20 \mu \mathrm{M}$ Pibrentasvir was added, exonuclease activity was substantially inhibited as shown by the reduced intensities of the fragmentation peaks and increased peak heights of the intact RNAs (c, f). Thus, the SARS-CoV-2 exonuclease activity is significantly inhibited by Pibrentasvir to prevent nucleotide excision from both Sofosbuvir and Remdesivir terminated RNA. Similarly, Pibrentasvir substantially reduced excision of the nucleotide analogues derived from Favipiravir (Fig. S-4), Molnupiravir (NHC, $\mathrm{N}^{4}$-hydroxycytidine, Fig. S-5) and AT-527 (Fig. S-6) from the 3' terminus of RNA. In terms of base pairing, Favipiravir-RTP acts like a G or $\mathrm{A}^{23}$, NHC-TP behaves like a C or $\mathrm{U}^{24}$ and AT-9010 (the active triphosphate form of AT-527) is a $\mathrm{G}$ analogue ${ }^{25}$. As expected, Favipiravir-RTP was incorporated into RNA opposite $\mathrm{C}$ or $\mathrm{U}$ in the template strand (Fig. S-4), NHC-TP was incorporated opposite A or G (Fig. S-5), and AT-9010 was incorporated opposite C (Fig. S-6) in the template by the RdRp complex (nsp12/nsp7/nsp8). In all cases, the incorporated nucleotide analogue along with the adjacent 6-7 nucleotides were efficiently removed following incubation with the exonuclease complex (nsp14/nsp10) (Fig. S-4b, e; S-5e, h; S-6e). However, in the presence of $20 \mu \mathrm{M}$ Pibrentasvir, this exonuclease activity was largely abrogated (Fig. S-4c, f; S-5f, i; S-6f).

The nucleotide inhibitor Remdesivir exhibits delayed termination during the polymerase reaction, which has been suggested as one of the possible mechanisms for inhibiting replication of SARS$\mathrm{CoV}-2^{14,15}$. However, if the exonuclease is able to remove the incorporated nucleotides including Remdesivir from this delayed termination RNA product, then the inhibitory activity of Remdesivir for SARS-CoV-2 will be reduced. Therefore, we investigated whether Pibrentasvir is efficient in preventing the excision of Remdesivir from delayed Remdesivir terminated RNA through inhibiting SARS-CoV-2 exonuclease activity. In Fig. 5, the RNA product displaying delayed Remdesivir (R) termination (sequence shown at the top of the figure) was incubated with the SARS-CoV-2 
nsp14/nsp10 complex at $37{ }^{\circ} \mathrm{C}$ for $15 \mathrm{~min}$ in the absence (e) and presence of $20 \mu \mathrm{M}$ of Pibrentasvir (f). MALDI-TOF MS analysis of the intact RNAs (d) and the products of the exonuclease reactions (e, f) was performed. In the absence of Pibrentasvir, exonuclease activity caused cleavage of 1-11 nucleotides from the 3'-end of the RNA as shown by the lower molecular weight fragments (e). When $20 \mu \mathrm{M}$ Pibrentasvir was added, exonuclease activity was inhibited as shown by the reduced intensities of the fragmentation peaks and increased peak of the intact RNAs (f, 9807 Da). In Fig. 5 (a, b, c), a comparison experiment was performed with natural RNA, which produced essentially identical results. Therefore, these results indicated that the SARS-CoV-2 exonuclease activity is also significantly inhibited by Pibrentasvir to prevent nucleotide excision from RNA with delayed termination by Remdesivir.

We previously showed that Tenofovir diphosphate (Tfv-DP) can be incorporated into RNA as an obligate terminator by the SARS-CoV-2 RdRp complex ${ }^{11}$. As an acyclic nucleotide, Tfv-DP lacks a normal sugar ring configuration, and thus we reasoned that it is less likely to be recognized by SARS-CoV-2 exonuclease. We therefore investigated whether $\mathrm{Tfv}$ can be removed by the exonuclease from the extended RNA. As shown in Fig. 6, Tfv-DP was incorporated into an RNA loop primer to produce the Tfv-terminated RNA (d). When incubation with the SARS-CoV-2 nsp14/nsp10 complex was carried out at $37^{\circ} \mathrm{C}$ for $15 \mathrm{~min}$, minimal cleavage was observed for Tfvterminated RNA (e) relative to RNA extended with a natural A nucleotide (b), where there was substantial cleavage. When $20 \mu \mathrm{M}$ Pibrentasvir was added, exonuclease activity was almost completely eliminated as shown by almost no fragmentation peaks and the essentially intact RNA peak (f). To directly compare the exonuclease resistance activities, we incubated both the RNA terminated with Tfv-DP and the natural RNA terminated with the A nucleotide in the same reaction tube, and compared the patterns obtained by MALDI-TOF MS (Fig. S-7). Because these two RNAs have different molecular weights, it was straightforward to determine whether the resulting cleavage products were derived from the Tfv- or the A-terminated RNA. The results shown in Figs. 6 and S-7 demonstrate that Tenofovir, once incorporated into RNA, is mostly resistant to excision by the SARS-CoV-2 exonuclease. 


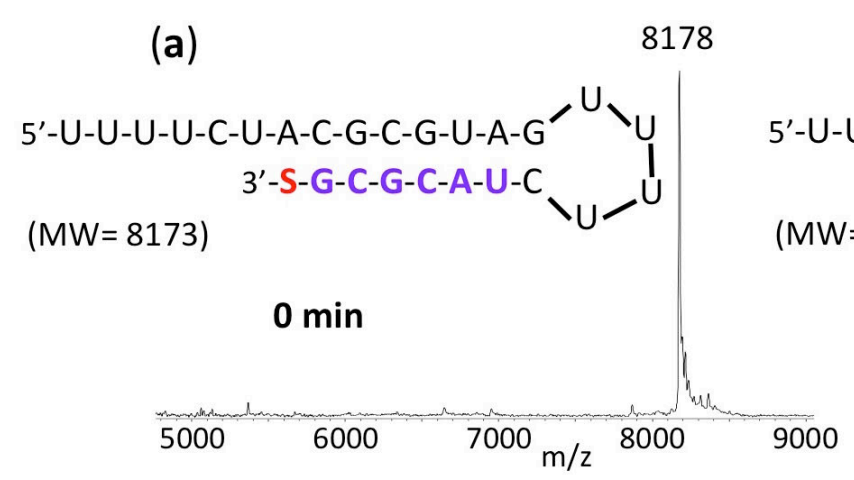

(b)

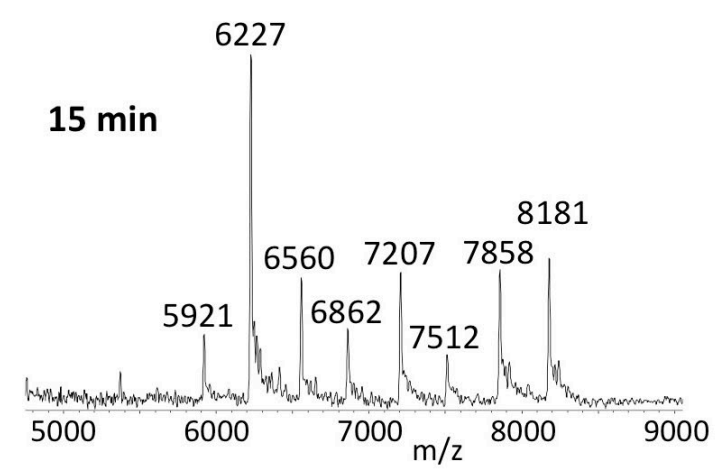

(c) + Pibrentasvir $(20 \mu \mathrm{M})$

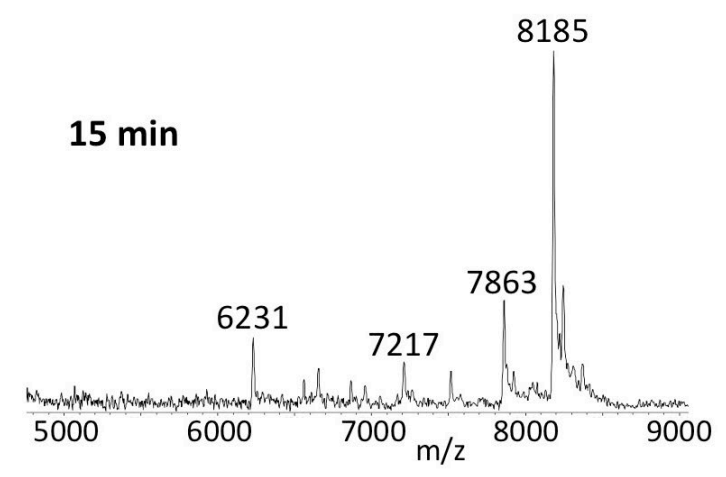

(d)

8209

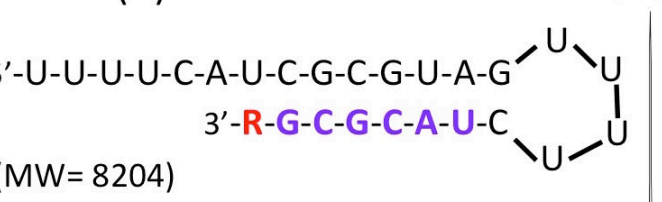

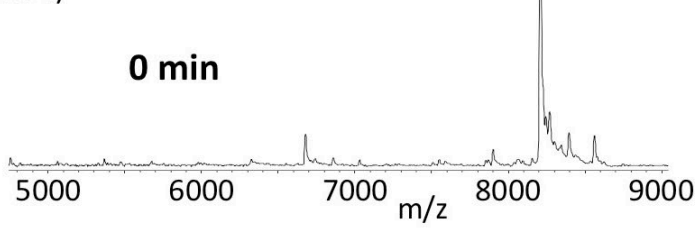

(e)
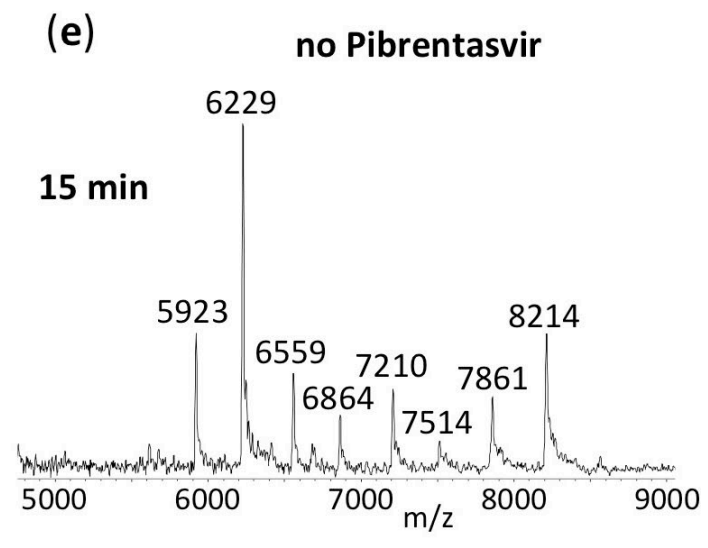

(f) + Pibrentasvir $(20 \mu \mathrm{M})$

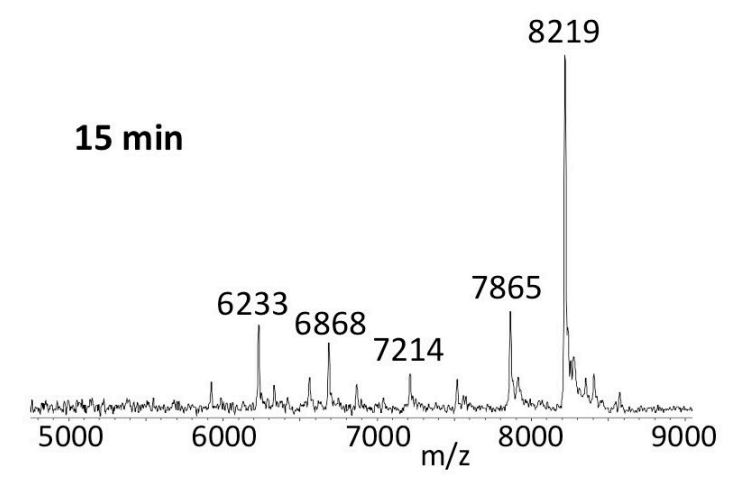

Fig. 4 Inhibition of SARS-CoV-2 exonuclease activity by Pibrentasvir for Sofosbuvir (S) and Remdesivir (R) terminated RNA. A mixture of $500 \mathrm{nM}$ RNAs (sequences shown at the top of the figure) and $50 \mathrm{nM}$ SARS-CoV-2 pre-assembled exonuclease complex (nsp14/nsp10) were incubated in buffer solution at $37^{\circ} \mathrm{C}$ for $15 \mathrm{~min}$ in the absence (b, e) and presence of $20 \mu \mathrm{M}$ Pibrentasvir (c, f). The intact RNAs (a, d) and the products of the exonuclease reactions (b-f) were analyzed by MALDI-TOF MS. The signal intensity was normalized to the highest peak. In the absence of Pibrentasvir, exonuclease activity caused nucleotide cleavage from the 3'-end of the RNA as shown by the lower molecular weight fragments corresponding to cleavage of 1-7 nucleotides $(b, e)$. When $20 \mu \mathrm{M}$ Pibrentasvir was added, exonuclease activity was reduced as shown by the reduced intensities of the fragmentation peaks and increased intact RNA peaks (c, f). 

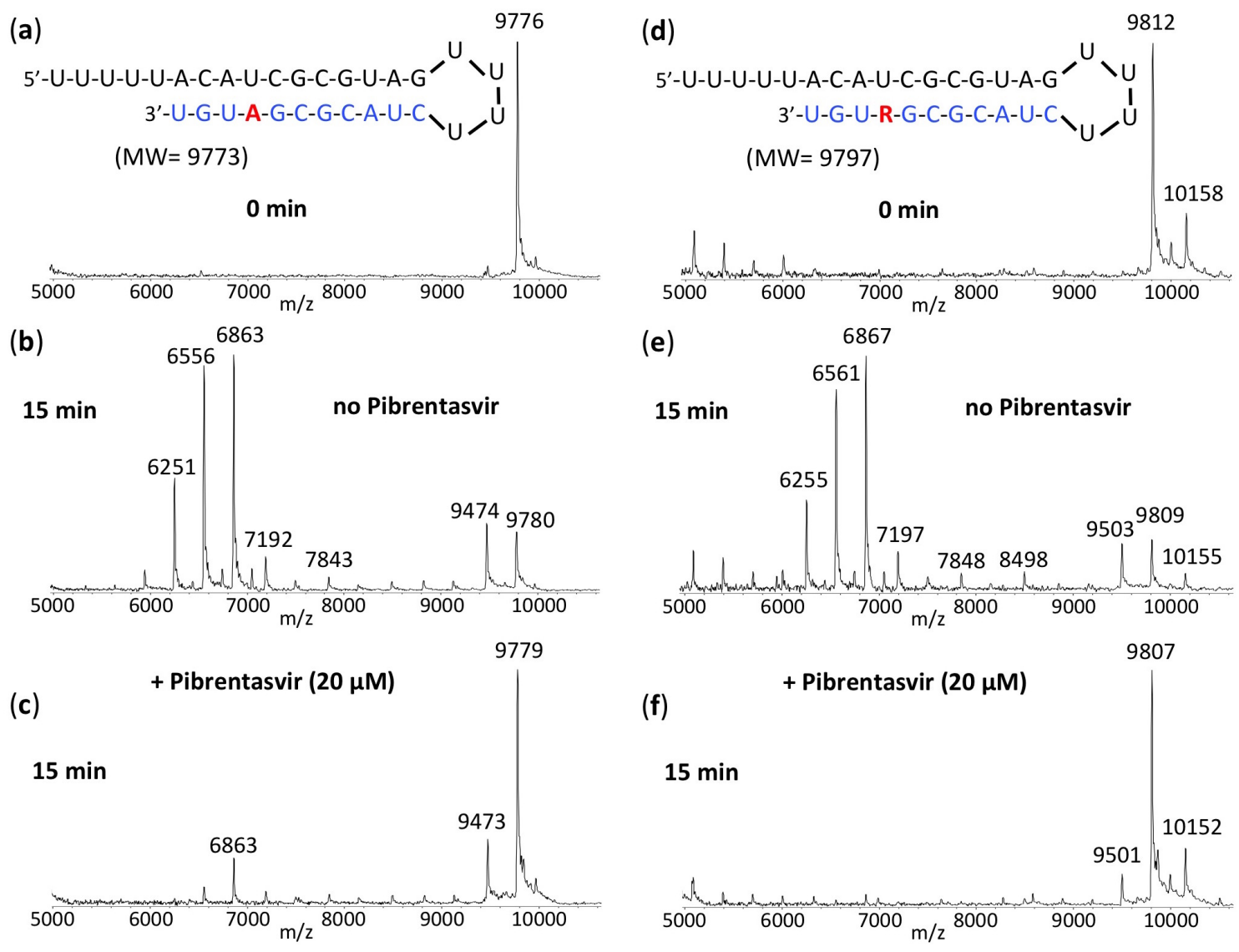

Fig. 5 Inhibition of SARS-CoV-2 exonuclease activity by Pibrentasvir for natural RNA and RNA with delayed termination by Remdesivir (R). A mixture of $400 \mathrm{nM}$ RNAs (sequences shown at the top of the figure) and $50 \mathrm{nM}$ SARS-CoV-2 pre-assembled exonuclease complex (nsp14/nsp10) were incubated in buffer solution at $37^{\circ} \mathrm{C}$ for $15 \mathrm{~min}$ in the absence (b, e) and presence of $20 \mu \mathrm{M}$ Pibrentasvir (c, f). The intact RNAs (a, d) and the products of the exonuclease reactions $(b, c, e, f)$ were analyzed by MALDI-TOF MS. The signal intensity was normalized to the highest peak. The peak at $9776 \mathrm{Da}$ corresponds to the intact RNA (9773 Da expected) and the peak at $9812 \mathrm{Da}$ corresponds to the intact Remdesivir delayed RNA termination product (9797 $\mathrm{Da}$ expected). The small peak at $10158 \mathrm{Da}$ corresponds to mismatched incorporation of an additional G; this is likely due to the low fidelity of SARS-CoV-2 RdRp ${ }^{11}$. In the absence of Pibrentasvir, exonuclease activity caused nucleotide cleavage from the 3 '-end of the RNA as shown by the lower molecular weight fragments corresponding to cleavage of 1-11 nucleotides (b, e). When $20 \mu \mathrm{M}$ Pibrentasvir was added, exonuclease activity was reduced as shown by the reduced intensities of the fragmentation peaks and increased intact RNA peaks $(c, f)$. 

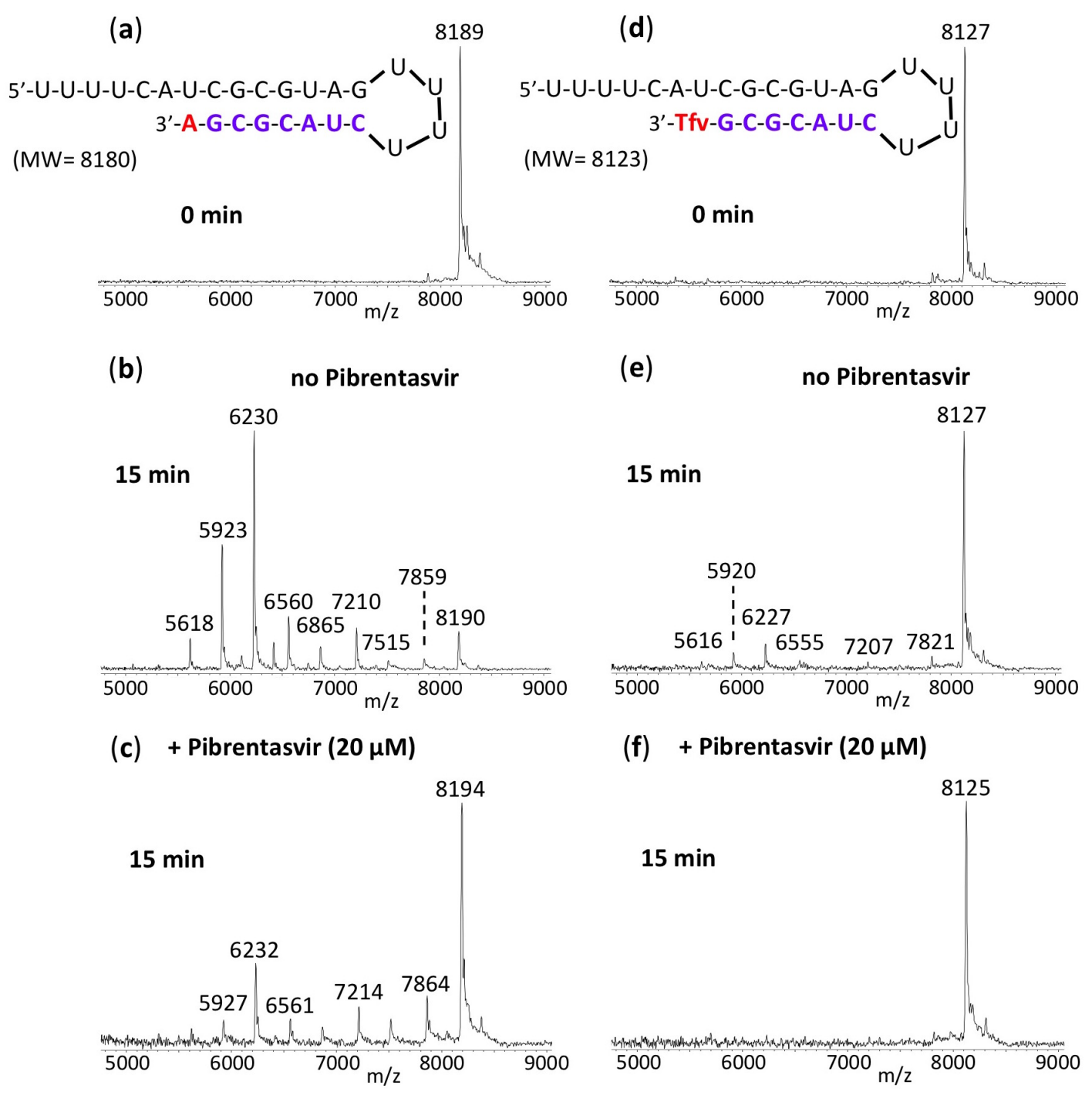

(c) + Pibrentasvir $(20 \mu \mathrm{M})$

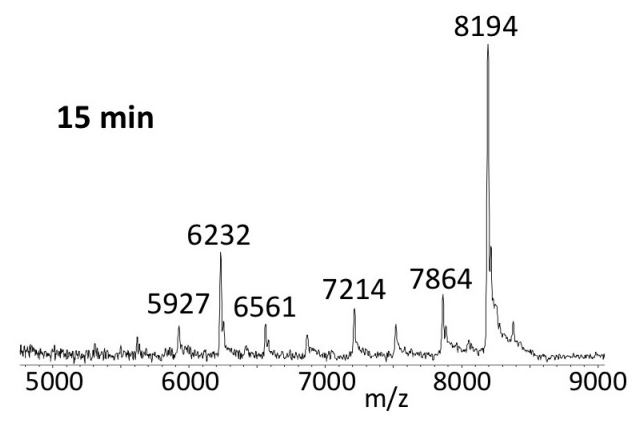

Fig. 6 Inhibition of SARS-CoV-2 exonuclease activity by Pibrentasvir for natural RNA and Tenofovir (Tfv) terminated RNA. A mixture of $500 \mathrm{nM}$ RNAs (sequences shown at the top of the figure) and $50 \mathrm{nM}$ SARS-CoV-2 pre-assembled exonuclease complex (nsp14/nsp10) were incubated in buffer solution at $37^{\circ} \mathrm{C}$ for $15 \mathrm{~min}$ in the absence (b, e) and presence of $20 \mu \mathrm{M}$ Pibrentasvir (c, f). The intact RNAs (a, d) and the products of the exonuclease reactions (b-f) were analyzed by MALDI-TOF MS. The signal intensity was normalized to the highest peak. In the absence of Pibrentasvir, exonuclease activity caused nucleotide cleavage from the 3'-end of the natural RNA as shown by the lower molecular weight fragments corresponding to cleavage of 1-8 nucleotides (b). However, for Tfv terminated RNA, only minor cleavage was observed (e). When $20 \mu \mathrm{M}$ Pibrentasvir was added, exonuclease activity was reduced as shown by the reduced intensities of the fragmentation peaks and increased peak height of the intact RNA (c, f). 
SARS-CoV-2 susceptibility to inhibition by immediate/delayed RNA chain terminators and error-prone nucleotide analogues is enhanced in Calu-3 cells by HCV NS5A inhibitors that inhibit the exonuclease.

The prodrugs of RdRp inhibitors are activated by cellular enzymes to the active triphosphates. Therefore, the above enzymatic results need to be further evaluated at the cellular level, as interference in any of the activation pathways may impact the synergistic inhibitory activity described in the enzymatic assays. We evaluated if SARS-CoV-2's in vitro susceptibility to clinically approved RdRp prodrugs could be enhanced by combining them with HCV NS5A inhibitors, which were shown to inhibit the exonuclease activity above. Calu-3 cells, which recapitulate the type II pneumocytes, cells destroyed in the course of severe COVID-19 26,27 , were infected at a MOI of 0.1 and treated with the drugs under investigation alone or in combination. After 2-3 days, culture supernatant was harvested and titered in VeroE6 cells to quantify the infectious viruses as plaque forming units (PFU). The NS5A inhibitors Pibrentasvir, Ombitasvir and Daclatasvir inhibited SARS-CoV-2 replication in a dose-dependent manner (Fig. S-8), but with lower potencies than Remdesivir (RDV) (Table S-1). Nevertheless, anti-SARS-CoV-2 potencies of the HCV NS5A inhibitors were 10 times higher compared to other repurposed RdRp inhibitors, such as Sofosbuvir, Tenofovir and Favipiravir (Table S-1).

To produce clinical benefit, inhibition of SARS-CoV-2 exonuclease by NS5A inhibitors need to enhance anti-coronavirus activity of RdRp inhibitors by orders of magnitude. Thus, Sofosbuvir and Tenofovir (immediate terminators), RDV (a delayed chain terminator) and Favipiravir (an errorprone nucleotide analogue) were tested at various concentrations along with the $\mathrm{EC}_{25}$ of Pibrentasvir $(0.1 \mu \mathrm{M})$, Ombitasvir $(0.1 \mu \mathrm{M})$ and Daclatasvir $(0.5 \mu \mathrm{M})$ (Table 1 and Fig. 7). Pibrentasvir improved RDV's efficacy at the $90 \%$ and $99 \%$ levels 6- and 3-fold, respectively (Table 1). Moreover, Pibrentasvir increased the potency of Favipiravir and Tenofovir 10-fold (Table 1). Importantly Favipiravir's $\mathrm{EC}_{90}$ value becomes lower than the threshold of plasma exposure ${ }^{28}$ in the presence of Pibrentasvir (Table 1). Ombitasvir enhanced RDV's and Tenofovir's activity similarly to Pibrentasvir (Table 1). For Favipiravir, Ombitasvir resulted in a $2-\log _{10}$ inhibition of SARS-CoV-2, reducing virus replication by 99\% (Table 1 and Fig. 7D). Daclatasvir enhanced Sofosbuvir and Tenofovir potencies 10 -fold at $\mathrm{EC}_{50}$, but only modestly increased RDV's efficacy at $\mathrm{EC}_{90}$ (Table 1). In the presence of Daclatasvir, Favipiravir's virus inhibitory efficacy at the $90 \%$ level was achievable (Table 1). These results demonstrate that the anti-exonuclease effect of NS5A inhibitors consistently enhanced the potency of prodrugs of the nucleotide analogues in the cell culture system. 

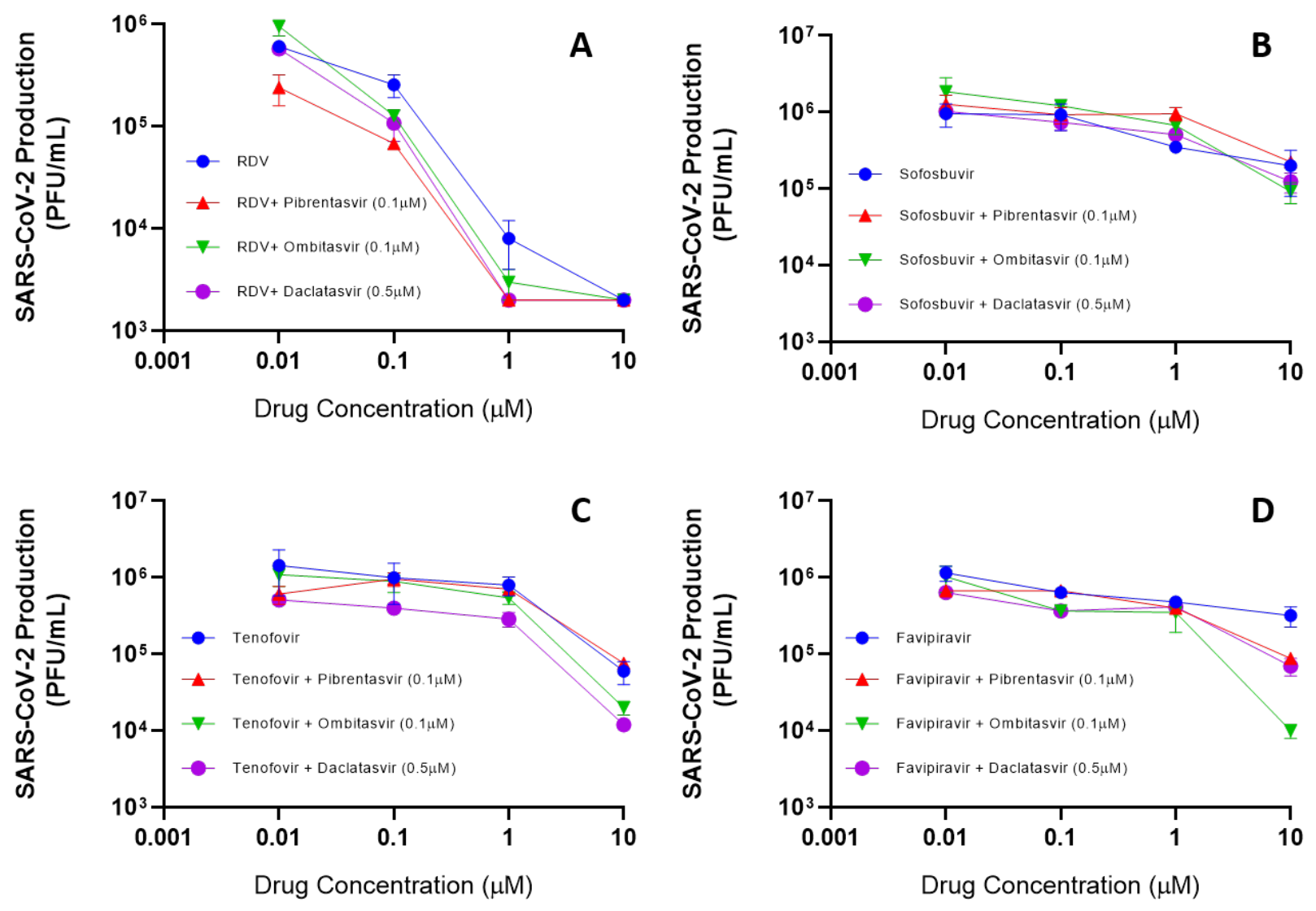

Fig. 7 Antiviral activity of combinations of SARS-CoV-2 polymerase and exonuclease inhibitors. Calu-3 cells, at a density of $5 \times 10^{5}$ cells/well in 48 -well plates, were infected with SARS-CoV-2 at a MOI of 0.1 , for $1 \mathrm{~h}$ at $37^{\circ} \mathrm{C}$. An inoculum was removed and cells were washed and incubated with fresh DMEM containing 2\% FBS and the indicated concentration of Remdesivir (RDV) (A), Sofosbuvir (B), Tenofovir (C), and Favipiravir (D), alone and in combination with the HCV NS5A inhibitors. Supernatants were assessed after 48-72 h. Viral replication in the culture supernatant was measured as PFU/mL by titering in VeroE6 cells. Results are displayed as virus titers. The data represent means \pm SEM of three independent experiments. 
Table 1 In vitro pharmacological parameters of potency and efficacy of combinations of RdRp and HCV NS5A inhibitors on SARS-CoV-2 replication in Calu-3 cells

\begin{tabular}{|l|c|c|c|c|c|c|}
\hline & \multicolumn{2}{c|}{ EC $_{\mathbf{5 0}}$} & \multicolumn{2}{c|}{ EC $_{90}$} & \multicolumn{2}{c|}{ EC $_{99}$} \\
\hline Drug & mean & SEM & mean & SEM & mean & SEM \\
\hline Remdesivir (RDV) & 0.09 & 0.002 & 0.4 & 0.03 & 1.1 & 0.2 \\
\hline Sofosbuvir & 6.2 & 0.3 & ND & ND & ND & ND \\
\hline Tenofovir & 4.3 & 2.1 & ND & ND & ND & ND \\
\hline Favipiravir & 7.8 & 1.2 & ND & ND & ND & ND \\
\hline Pibrentasvir & 0.7 & 0.2 & 4.2 & 0.6 & ND & ND \\
\hline RDV + Pibrentasvir $(0.1 \mu \mathrm{M})$ & 0.008 & 0.0009 & 0.07 & 0.03 & 0.3 & 0.09 \\
\hline Sofosbuvir + Pibrentasvir $(0.1 \mu \mathrm{M})$ & 8.3 & 0.5 & ND & ND & ND & ND \\
\hline Tenofovir + Pibrentasvir $(0.1 \mu \mathrm{M})$ & 0.5 & 0.05 & 8 & 1.5 & ND & ND \\
\hline Favipiravir + Pibrentasvir $(0.1 \mu \mathrm{M})$ & 0.5 & 0.03 & 8 & 0.5 & ND & ND \\
\hline Ombitasvir & 0.4 & 0.05 & 3.3 & 0.5 & ND & ND \\
\hline RDV + Ombitasvir $(0.1 \mu \mathrm{M})$ & 0.008 & 0.0003 & 0.01 & 0.05 & 0.5 & 0.2 \\
\hline Sofosbuvir + Ombitasvir $(0.1 \mu \mathrm{M})$ & 6 & 1.3 & 9 & 5 & ND & ND \\
\hline Tenofovir + Ombitasvir $(0.1 \mu \mathrm{M})$ & 0.8 & 0.07 & 7 & 1.6 & 8.9 & 0.4 \\
\hline Favipiravir + Ombitasvir $(0.1 \mu \mathrm{M})$ & 0.15 & 0.04 & 8 & 0.4 & 9.5 & 0.4 \\
\hline Daclatasvir & 0.7 & 0.08 & 3.8 & 1.2 & ND & ND \\
\hline RDV + Daclatasvir $(0.5 \mu \mathrm{M})$ & 0.008 & 0.0006 & 0.1 & 0.06 & 0.5 & 0.1 \\
\hline Sofosbuvir + Daclatasvir $(0.5 \mu \mathrm{M})$ & 0.6 & 0.05 & 9 & 0.2 & ND & ND \\
\hline Tenofovir + Daclatasvir $(0.5 \mu \mathrm{M})$ & 0.01 & 0.004 & 6 & 1.2 & 7.5 & 0.5 \\
\hline Favipiravir + Daclatasvir $(0.5 \mu \mathrm{M})$ & 0.12 & 0.05 & 8 & 0.5 & ND & ND \\
\hline
\end{tabular}

\section{Discussion}

We previously demonstrated that the FDA approved HCV NS5A inhibitors, Daclatasvir and Velpatasvir, and to a lesser extent the NS5A inhibitors Elbasvir and Ledipasvir, can inhibit the SARS-CoV-2 exonuclease ${ }^{29}$. Of particular interest, Daclatasvir and Velpatasvir inhibit both the SARS-CoV-2 polymerase and exonuclease ${ }^{20,21}$. Here, we showed that two additional NS5A inhibitors, Pibrentasvir and Ombitasvir, also inhibit the exonuclease, and have the highest inhibitory activity based on our molecular assay. These compounds are predicted to interfere with the binding of the $\mathrm{Mg}^{++}$ion with the 3' terminus of the RNA in the active site of the exonuclease (nsp14). The $\mathrm{Mg}^{++}$ion coordinates amino acid residues Asp-90, Glu-92, Glu-191 and Asp-273 and the 3' terminus of the RNA. Because the NS5A inhibitors interfere with this coordination, they are likely to prevent nucleotide excision from the RNA (Fig. 2). A recent in silico modeling study has suggested that Ritonavir also binds to the active site of nsp14, which led the authors to the prediction that Ritonavir may inhibit exonuclease activity ${ }^{30}$. We have experimentally shown (Fig. S-9) that Ritonavir and Lopinavir, HIV protease inhibitors that make up the combination drug Kaletra, inhibit the SARSCoV-2 exonuclease in a concentration-dependent manner, but with less potency than Pibrentasvir and Ombitasvir. Kaletra has gone through extensive clinical trials for COVID-19 but shows limited efficacy by itself ${ }^{31,32}$. Recently, using a digital drug development approach combining artificial 
intelligence and experimental validation to screen drug combinations for potential combination therapy against SARS-CoV-2, it was determined that one of the most effective potential combinations was Remdesivir, Ritonavir and Lopinavir ${ }^{33}$. This synergy of Ritonavir and Lopinavir with Remdesivir may be due to the exonuclease inhibitory activity of these two drugs (Fig. S-9).

NS5A inhibitors enhance the antiviral activity of RDV, Tenofovir, Sofosbuvir and Favipiravir, likely due to their inhibition of exonuclease activity. The combinations of drugs described here, especially the orally available antivirals, have the potential to progress to COVID-19 clinical trials. We show that by combining Pibrentasvir or Ombitasvir with Remdesivir, Sofosbuvir, Tenofovir or Favipiravir, higher inhibitory activity for SARS-CoV-2 was achievable at lower doses, bringing the nucleotides' pharmacological parameters more in line with their pharmacokinetic exposures ${ }^{28,34,35}$. The nucleotide inhibitors Remdesivir and Sofosbuvir are incorporated into the replicating RNA to inhibit further polymerase reaction, but they are rapidly removed by the exonuclease in the absence of exonuclease inhibitors such as Pibrentasvir and Ombitasvir. However, in the presence of these inhibitors, any nucleotide inhibitors incorporated into the RNA will not be rapidly excised by the exonuclease and their inhibitory effect on overall viral RNA replication will be substantially enhanced. Recently, the coronavirus exonuclease has also been shown to promote viral recombination which can increase the emergence of novel strains ${ }^{36}$, providing additional incentive for targeting this enzyme with antivirals.

Previously, Baddock et al. screened a series of molecules for SARS-CoV-2 exonuclease activity, including Ebselen and Disulfiram, with Ebselen having the higher inhibitory activity ${ }^{37}$. Ebselen and Disulfiram have multiple additional SARS-CoV-2 protein targets and Chen et al. used a combination of Ebselen/Disulfiram with Remdesivir to demonstrate a small synergistic inhibitory effect on viral replication $^{38}$. Recently, it has been reported that $\mathrm{HCV}$ nsp3/4A protease inhibitors that inhibit the SARS-CoV-2 papain-like protease synergize with Remdesivir to suppress viral replication in cell culture $^{39}$. In our investigation, by focusing on combinations of clinically approved drugs used during routine treatment of $\mathrm{HIV} / \mathrm{HCV} /$ influenza infected individuals, we discovered significant synergistic combinations of drugs that inhibit both the SARS-CoV-2 polymerase and exonuclease activities.

Favipiravir (6-fluoro-3-hydroxy-2-pyrazinecarboxamide, Fig. 1c), a drug used to treat influenza, has been investigated for treatment of COVID-19 $9^{40,41}$. It has a modified nucleobase that can base pair with both $\mathrm{C}$ and $\mathrm{U}$. Favipiravir is converted to the active triphosphate form, Favipiravirribofuranosyl-5'-triphosphate (Favipiravir-RTP), by a cellular enzyme, hypoxanthine guanine phosphoribosyltransferase and cellular kinases (Fig. S-10). Favipiravir-RTP acts as a viral RNA polymerase inhibitor, with great potential in the treatment of a wide variety of RNA virus infections, including different strains of influenza ${ }^{42,43}$. Favipiravir-RTP is incorporated into the growing RNA chain by the SARS-CoV-2 RdRp complex and causes C-to-U and G-to-A transitions. In a more recent study, Favipiravir was found to exhibit antiviral activity against SARS-CoV-2 via a combination of mechanisms, including slowing RNA synthesis, initiation of delayed chain termination, and lethal mutagenesis ${ }^{23,40}$. The 3-D structure of the SARS-CoV-2 RdRp complex (nsp12, nsp7 and nsp8) with the RNA substrate and Favipiravir-RTP was determined, providing insight into the mode of action of this drug ${ }^{44,45}$. Here, we demonstrated that the excision of Favipiravir from the 3' end of RNA by the SARS-CoV-2 exonuclease complex is greatly reduced in the presence of Pibrentasvir (Fig. S-4). Since Pibrentasvir and Ombitasvir inhibited the SARS-CoV2 exonuclease proofreading activity, when these drugs are combined with Favipiravir, it is more likely that Favipiravir-induced mutations will persist and jeopardize viral replication. This is 
supported by our cell culture virus inhibition data indicating that the combination of Favipiravir with Ombitasvir resulted in a $2-\log _{10}$ inhibition, reducing virus replication by $99 \%$ (Table 1 ).

$\mathrm{N}^{4}$-hydroxycytidine (NHC, Fig. 1e) has been shown to inhibit SARS-CoV-2 and other coronaviruses in mice and human airway epithelial cells ${ }^{24}$. NHC is incorporated opposite either $A$ or $G$ in the template strand. Molnupiravir, which is activated to the triphosphate form (NHC-TP) by cellular enzymes, is currently in a COVID-19 clinical trial ${ }^{46}$. Here, we showed that the excision of NHC from the 3' end of RNA by the SARS-CoV-2 exonuclease complex is substantially reduced in the presence of Pibrentasvir (Fig. S-5).

AT-527 (Fig. 1f), a prodrug of a guanosine nucleotide analogue with both the base and phosphate group masked, is converted by cellular enzymes to the active triphosphate form, 2'-fluoro-2'-methyl guanosine-5' -triphosphate (AT-9010, $\mathrm{G}_{\mathrm{fm}}$-triphosphate) ${ }^{25}$. The active triphosphate form of AT-527 is structurally similar to that of Sofosbuvir with the only difference being that it has a guanine base in place of uracil. AT-527 inhibits SARS-CoV-2 by targeting both the RdRp activity and the Nidovirus RdRp-associated nucleotidyltransferase (NiRAN) activity of nsp12, which are essential for viral RNA replication and transcription ${ }^{47}$. AT-527 is currently in COVID-19 clinical trials. Here, we demonstrated that the excision of AT-527 from the 3' end of RNA by the SARS-CoV-2 exonuclease is largely abrogated by the exonuclease inhibitor Pibrentasvir (Fig. S-6).

Additionally, we have shown that when Tenofovir is incorporated into RNA, it is mostly resistant to excision by the SARS-CoV-2 exonuclease, which might be explained by the fact that it is an acyclic nucleotide analogue, interfering with its recognition by the exonuclease. The addition of Pibrentasvir almost completely protected the Tenofovir terminated RNA from excision by exonuclease. The combination of NS5A inhibitors with Tenofovir also substantially increased the virus inhibition efficiency in cell culture (Table 1).

Our results from the molecular enzymatic assays and the virus cell culture inhibitory data both indicate that the use of inhibitors of the viral RdRp and the exonuclease proofreader as a combination therapy is expected to have enhanced efficacy in treating COVID-19. This combination approach may allow two- $\log _{10}$ reduction of virus replication using concentrations consistent with approved plasma exposure. We anticipate that the various nucleotide analogue inhibitors of the SARS-CoV-2 RdRp that are in clinical trials for COVID-19 will benefit from the addition of exonuclease inhibitors.

\section{Methods}

The RdRp of SARS-CoV-2, referred to as nsp12, and its two protein cofactors, nsp7 and nsp8, shown to be required for the processive polymerase activity of nsp12, were cloned and purified as described $^{11,20}$. The $3^{\prime}$-exonuclease, referred to as nsp14, and its protein cofactor, nsp10, were cloned and expressed based on the SARS-CoV-2 genome sequence. Remdesivir triphosphate (RDV-TP) and NHC triphosphate were purchased from MedChemExpress (Monmouth Junction, NJ), Sofosbuvir triphosphate (SOF-TP) was purchased from Sierra Bioresearch (Tucson, AZ), Tenofovir diphosphate (Tfv-DP) was purchased from Alfa Chemistry (Ronkonkoma, NY), and Favipiravir triphosphate and AT-9010 were purchased from NuBlocks LLC (Oceanside, CA). Pibrentasvir was purchased from Cayman Chemical Company (Ann Arbor, MI) and Ombitasvir, Daclatasvir, 
Ritonavir and Lopinavir were purchased from Selleckchem (Houston, TX). UTP, GTP and ATP were purchased from Fisher Scientific. The RNA oligonucleotides (template-loop-primers) were purchased from Dharmacon (Horizon Discovery, Lafayette, $\mathrm{CO}$ ).

\section{Expression and purification of the SARS-CoV-2 exonuclease nsp14/nsp10 complex.}

The 3'-exonuclease, referred to as nsp14, and its protein cofactor, nsp10, were cloned and expressed based on the SARS-CoV-2 genome sequence. The pRSFDuet-1 plasmids (Novagen) encoding SARS-CoV-2 nsp14 or nsp10 engineered with an N-terminal His-SUMO tag were prepared as follows: SARS-CoV-2 RNA isolated from the supernatant of SARS-CoV-2-infected Vero E6 cells was provided by Benjamin R. tenOever ${ }^{48}$. The portion of the RNA encoding nsp10 and nsp14 was reverse transcribed into cDNA using gene-specific primers (see below) and SuperScript III Reverse Transcriptase (Thermo Fisher). The nsp10 and nsp14 coding sequences were PCR amplified using forward and reverse gene specific primers flanked by BamHI and XhoI recombination sites, respectively. PCR products were digested with BamHI and XhoI and subsequently ligated into BamHI and XhoI-digested pRSFDuet-His6-sumo vector and sequence verified. pRSFDuet-His6sumo is a modified pRSFDuet-1 vector (Novagen) bearing an N-terminal His6-SUMO-tag cleavable by the ubiquitin-like protease (ULP1).

List of PCR primers used:

NSP14_pRSF_BamHI_fw

CGCGGATCCGCTGAAAATGTAACAGGACTCTTTAAA

NSP14_pRSF_XhoI_rev

CCCGCTCGAGCGGTCACTGAAGTCTTGTAAAAGTGTTCCAGAGG

RT_primer_NSP14

TTCTTGGCTATGTCAGTCATAGAACAAAC

NSP10_pRSF_BamHI_fw

CGCGGATCCGCTGGTAATGCAACAGAAGTGCCTGCC

NSP10_pRSF_XhoI_rev

CCCGCTCGAGCGGTCACTGAAGCATGGGTTCGCGGAGTTGATC

RT_primer_NSP10

GATGTTGATATGACATGGTCGTAACAGC

The nsp14 and nsp10 proteins were expressed in Escherichia coli BL21-CodonPlus(DE3)-RIL (Stratagene). The bacteria were grown in Luria-Bertani medium supplemented with $50 \mathrm{mg} / \mathrm{mL}$ kanamycin at $37{ }^{\circ} \mathrm{C}$ to an $\mathrm{OD} 600$ of 0.6 , and induced with $0.4 \mathrm{mM}$ isopropyl $\beta$-D-1thiogalactopyranoside and $50 \mu \mathrm{M} \mathrm{ZnCl} 2$ overnight at $18{ }^{\circ} \mathrm{C}$. Cells were collected via centrifugation at 5,000 $\times \mathrm{g}$ and equal volumes of the nsp14 and nsp10 bacterial cells were then mixed for nsp14nsp10 protein complex purification. The cells were lysed via sonication in Lysis Buffer $(500 \mathrm{mM}$ $\mathrm{NaCl}, 20 \mathrm{mM}$ imidazole, $20 \mathrm{mM}$ Tris- $\mathrm{HCl}, \mathrm{pH}$ 8.0, $1 \mathrm{mM}$ phenylmethylsulfonyl fluoride). After 
centrifugation at 40,000 $\times \mathrm{g}$, the supernatant was loaded onto $5 \mathrm{~mL}$ Nickel Sepharose 6 fast flow resins (GE Healthcare) in a gravity flow column. The target protein was eluted using Lysis Buffer supplemented with $500 \mathrm{mM}$ imidazole. The eluted protein was incubated with ULP1 (lab stock) during dialysis at $4{ }^{\circ} \mathrm{C}$ overnight against a buffer containing $20 \mathrm{mM}$ Tris-HCl, pH 7.5, $20 \mathrm{mM}$ imidazole, $150 \mathrm{mM} \mathrm{NaCl}, 100 \mu \mathrm{M} \mathrm{ZnCl}$, and $5 \mathrm{mM} \beta$-mercaptoethanol. Then the sample was loaded onto the HisTrap FF column (GE Healthcare) to remove the His-SUMO tag, and the flowthrough was collected. The target proteins were further purified through a Superdex200 10/300 gel filtration column (GE Healthcare) in a buffer containing $20 \mathrm{mM}$ HEPES, $\mathrm{pH} 7.4,150 \mathrm{mM} \mathrm{NaCl}, 1$ $\mathrm{mM} \mathrm{MgCl}$, and $1 \mathrm{mM}$ dithiothreitol. The fractions corresponding to the nsp14/nsp10 complex were detected by SDS-PAGE and collected. The protein sample was flash-frozen in liquid nitrogen and stored at $-80{ }^{\circ} \mathrm{C}$.

\section{Extension reactions with SARS-CoV-2 RNA-dependent RNA polymerase complex to produce Remdesivir (RDV) and Sofosbuvir (SOF) terminated RNAs.}

$10 \mu \mathrm{L}$ of $10 \mu \mathrm{M}$ RNA template-loop-primers (5'-UUUUCAUCGCGUAGUUUUCUACGCG-3' for RDV-TP extension; 5'-UUUUCUACGCGUAGUUUUCUACGCG-3' for SOF-TP extension) in $1 \times$ $\mathrm{RdRp}$ reaction buffer was annealed by heating to $75^{\circ} \mathrm{C}$ for $3 \mathrm{~min}$ and cooling to room temperature. 5 $\mu \mathrm{L}$ of $8 \mu \mathrm{M}$ RdRp complex (nsp12/nsp7/nsp8) ${ }^{11,20}$ in $1 \times$ reaction buffer was added to the annealed RNA template-loop-primer solution and incubated for an additional $10 \mathrm{~min}$ at room temperature. Finally, $5 \mu \mathrm{L}$ of a solution containing $0.2 \mathrm{mM}$ RDV-TP or $2 \mathrm{mM}$ SOF-TP in $1 \times$ reaction buffer was added and incubation was carried out for $2 \mathrm{~h}$ at $30^{\circ} \mathrm{C}$. The final concentrations of reagents in the 20 $\mu \mathrm{L}$ extension reactions were $2 \mu \mathrm{M}$ nsp12/nsp7/nsp8, $5 \mu \mathrm{M}$ RNA template-loop-primer, $50 \mu \mathrm{M}$ RDV-TP and $500 \mu \mathrm{M}$ SOF-TP. The $1 \times$ reaction buffer contains the following reagents: $10 \mathrm{mM}$ Tris- $\mathrm{HCl} \mathrm{pH} 8,10 \mathrm{mM} \mathrm{KCl}, 2 \mathrm{mM} \mathrm{MgCl} 2$ and $1 \mathrm{mM} \beta$-mercaptoethanol. Desalting of the reaction mixture was performed with an Oligo Clean \& Concentrator kit (Zymo Research) resulting in $~ 10$ $\mu \mathrm{L}$ purified aqueous RNA solutions. $1 \mu \mathrm{L}$ of each solution was subjected to MALDI-TOF MS (Bruker ultrafleXtreme) analysis. The remaining $\sim 9 \mu \mathrm{L}$ extended template-loop-primer solutions were used to test exonuclease activity.

\section{Extension reactions with Therminator II to produce AT-9010 terminated RNA}

The RNA template-loop primer (5'-UUUUCUCCGCGUAGUUUUCUACGCG-3') was annealed at $75^{\circ} \mathrm{C}$ for 3 minutes and cooled to room temperature for 25 minutes in $1 \times$ ThermoPol buffer before adding the other ingredients. The final $20 \mu \mathrm{L}$ mixture containing $5 \mu \mathrm{M}$ of the RNA template-loop primer, $250 \mu \mathrm{M}, 500 \mu \mathrm{M}$ or $1 \mathrm{mM} \mathrm{AT-9010,2} \mathrm{mM} \mathrm{MnCl}_{2}$ and 0.2 unit Therminator II in $1 \times$ ThermoPol buffer was incubated in a thermal cycler using the following protocol $\left(28\right.$ cycles of $45^{\circ} \mathrm{C}$ for $30 \mathrm{sec}, 55^{\circ} \mathrm{C}$ for $30 \mathrm{sec}, 65^{\circ} \mathrm{C}$ for $30 \mathrm{sec}$ ). Desalting of the reaction mixture was performed with an Oligo Clean \& Concentrator kit (Zymo Research) resulting in $\sim 10 \mu \mathrm{L}$ purified aqueous RNA solutions. $1 \mu \mathrm{L}$ of each solution was subjected to MALDI-TOF MS (Bruker ultrafleXtreme) analysis. The remaining $\sim 9 \mu \mathrm{L}$ extended template-loop-primer solutions were used to test exonuclease activity. 


\section{Extension reactions with SARS-CoV-2 RNA-dependent RNA polymerase to produce Favipiravir or NHC terminated RNAs}

$10 \mu \mathrm{L}$ of $10 \mu \mathrm{M}$ RNA template-loop-primers (5'- UUUUCAUCGCGUAGUUUUCUACGCG-3' for Fav-RTP extension opposite U; 5'-UUUUCACCGCGUAGUUUUCUACGCG-3' for Fav-RTP extension opposite C; 5'- UUUUCUACGCGUAGUUUUCUACGCG-3' for NHC-TP extension opposite A; 5'-UUUUCUGCGCGUAGUUUUCUACGCG-3' for NHC-TP extension opposite G) in $1 \times \operatorname{RdRp}$ reaction buffer was annealed by heating to $75{ }^{\circ} \mathrm{C}$ for $3 \mathrm{~min}$ and cooling to room temperature. $5 \mu \mathrm{L}$ of $12 \mu \mathrm{M}$ RdRp complex $(\mathrm{nsp} 12 / \mathrm{nsp} 7 / \mathrm{nsp} 8)^{11,20}$ in $1 \times$ reaction buffer was added to the annealed RNA template-loop-primer solution and incubated for an additional $10 \mathrm{~min}$ at room temperature. Finally, $5 \mu \mathrm{L}$ of a solution containing $\sim 2 \mathrm{mM}$ Fav-RTP or $0.4 \mathrm{mM} \mathrm{NHC}$ in $1 \times$ reaction buffer was added and incubation was carried out for $3 \mathrm{~h}$ at $30{ }^{\circ} \mathrm{C}$. The final concentrations of reagents in the $20 \mu \mathrm{L}$ extension reactions were $3 \mu \mathrm{M}$ nsp12/nsp7/nsp8, $5 \mu \mathrm{M}$ RNA template-loopprimer and $\sim 500 \mu \mathrm{M}$ Fav-RTP or $100 \mu \mathrm{M}$ NHC-TP. The $1 \times$ reaction buffer contains the following reagents: $10 \mathrm{mM}$ Tris- $\mathrm{HCl} \mathrm{pH} 8,10 \mathrm{mM} \mathrm{KCl}, 2 \mathrm{mM} \mathrm{MgCl}$ and $1 \mathrm{mM} \beta$-mercaptoethanol. Desalting of the reaction mixture was performed with an Oligo Clean \& Concentrator kit (Zymo Research) resulting in $\sim 10 \mu \mathrm{L}$ purified aqueous RNA solutions. $1 \mu \mathrm{L}$ of each solution was subjected to MALDI-TOF MS (Bruker ultrafleXtreme) analysis. The remaining $\sim 9 \mu \mathrm{L}$ extended template-loopprimer solutions were used to test exonuclease activity.

\section{Extension reactions with reverse transcriptase to produce Tenofovir (Tfv) terminated RNAs.}

The RNA template-loop primer (5'-UUUUCAUCGCGUAGUUUUCUACGCG-3') was annealed at $75^{\circ} \mathrm{C}$ for 3 minutes and cooled to room temperature for 25 minutes before adding the remaining ingredients. The final $20 \mu \mathrm{L}$ mixture containing $5 \mu \mathrm{M}$ of the RNA template-loop primer, $500 \mu \mathrm{M}$ Tenofovir-DP and 200 units Superscript IV Reverse Transcriptase in $1 \times$ reaction buffer $(50 \mathrm{mM}$ Tris- $\mathrm{HCl}\left(\mathrm{pH} \mathrm{8.3)}, 4 \mathrm{mM} \mathrm{MgCl}_{2}, 10 \mathrm{mM} \mathrm{DTT}, 50 \mathrm{mM} \mathrm{KCl}\right)$ was incubated at $45{ }^{\circ} \mathrm{C}$ for $3 \mathrm{hr}$. Desalting of the reaction mixture was performed with an Oligo Clean \& Concentrator kit (Zymo Research) resulting in $\sim 10 \mu \mathrm{L}$ purified aqueous RNA solutions. $1 \mu \mathrm{L}$ of each solution was subjected to MALDI-TOF MS (Bruker ultrafleXtreme) analysis. The remaining $\sim 9 \mu \mathrm{L}$ extended templateloop-primer solutions were used to test exonuclease activity.

\section{Extension reactions with SARS-CoV-2 RNA-dependent RNA polymerase to produce Remdesivir (RDV) delayed terminated RNA.}

$10 \mu \mathrm{L}$ of $10 \mu \mathrm{M}$ RNA template-loop-primers (5'-UUUUUACAUCGCGUAGUUUUCUACGCG-3' for RDV-TP + UTP extension) in $1 \times \mathrm{RdRp}$ reaction buffer was first annealed by heating to $75^{\circ} \mathrm{C}$ for $3 \mathrm{~min}$ and cooling to room temperature. $5 \mu \mathrm{L}$ of $8 \mu \mathrm{M}$ RdRp complex (nsp12/nsp $7 / \mathrm{nsp} 8)^{11,20}$ in 1 $\times$ reaction buffer was added to the annealed RNA template-loop-primer solution and incubated for an additional $10 \mathrm{~min}$ at room temperature. $5 \mu \mathrm{L}$ of a solution containing $0.2 \mathrm{mM} \mathrm{RDV}-\mathrm{TP}$ and 0.2 mM UTP in $1 \times$ reaction buffer was added and incubation was carried out for $1 \mathrm{~h}$ at $30^{\circ} \mathrm{C}$. The final concentrations of reagents in the $20 \mu \mathrm{L}$ extension reactions were $2 \mu \mathrm{M}$ nsp $12 / \mathrm{nsp} 7 / \mathrm{nsp} 8,5 \mu \mathrm{M}$ RNA template-loop-primer, $50 \mu \mathrm{M}$ RDV-TP and $50 \mu \mathrm{M}$ UTP. The composition of $1 \times$ reaction buffer was as described above. Desalting of the reaction mixture was performed with an Oligo Clean \& Concentrator kit (Zymo Research) resulting in $\sim 10 \mu \mathrm{L}$ purified aqueous RNA solutions. $1 \mu \mathrm{L}$ of each solution was subjected to MALDI-TOF MS (Bruker ultrafleXtreme) analysis. The remaining $\sim 9 \mu \mathrm{L}$ extended template-loop-primer solutions were used for the second extension reaction. 
Then, $10 \mu \mathrm{L}$ of $\sim 16 \mu \mathrm{M}$ Remdesivir (R) and uridine extended RNA template-loop-primers (5'UUUUUACAUCGCGUAGUUUUCUACGCGRU-3' for second extension) in $1 \times$ RdRp reaction buffer was annealed by heating to $75^{\circ} \mathrm{C}$ for $3 \mathrm{~min}$ and cooling to room temperature. $5 \mu \mathrm{L}$ of $8 \mu \mathrm{M}$ RdRp complex (nsp12/nsp7/nsp8) ${ }^{11,20}$ in $1 \times$ reaction buffer was added to the annealed RNA template-loop-primer solution and incubated for an additional $10 \mathrm{~min}$ at room temperature. $5 \mu \mathrm{L}$ of a solution containing $80 \mu \mathrm{M}$ GTP and $80 \mu \mathrm{M}$ UTP in $1 \times$ reaction buffer was added and incubation was carried out for $1 \mathrm{~h}$ at $30^{\circ} \mathrm{C}$. The final concentrations of reagents in the $20 \mu \mathrm{L}$ extension reactions were $2 \mu \mathrm{M}$ nsp12/nsp7/nsp8, $\sim 4 \mathrm{M}$ RNA template-loop-primer, $20 \mu \mathrm{M}$ GTP and $20 \mu \mathrm{M}$ UTP. The composition of $1 \times$ reaction buffer was as described above. Desalting of the reaction mixture was performed with an Oligo Clean \& Concentrator kit (Zymo Research) resulting in $\sim 10$ $\mu \mathrm{L}$ purified aqueous RNA solutions. $1 \mu \mathrm{L}$ of each solution was subjected to MALDI-TOF MS (Bruker ultrafleXtreme) analysis. The remaining $\sim 9 \mu \mathrm{L}$ extended template-loop-primer solutions were used to test exonuclease activity.

\section{SARS-CoV-2 exonuclease reactions in the presence and absence of Pibrentasvir, Ombitasvir, Daclatasvir, Ritonavir and Lopinavir.}

The U-terminated RNA, A-terminated RNA, G-terminated RNA, the Remdesivir (RDV), Sofosbuvir (SOF), Favipiravir (Fav), $\mathrm{N}^{4}$-hydroxycytidine (Nhc), AT-9010 and Tenofovir (Tfv) terminated RNAs as well as the RDV delayed terminated RNA products from above, were annealed by heating to $75^{\circ} \mathrm{C}$ for $3 \mathrm{~min}$ and cooling to room temperature in $1 \times$ exonuclease reaction buffer. To a $14 \mu \mathrm{L}$ solution of $71.4 \mathrm{nM}$ exonuclease complex (nsp14/nsp10) in $1 \times$ exonuclease reaction buffer, $1 \mu \mathrm{L}$ of DMSO with or without various concentrations of Pibrentasvir was added and incubated for 15 min at room temperature. Then, $5 \mu \mathrm{L}$ of the annealed RNA $(2 \mu \mathrm{M})$ in $1 \times$ exonuclease reaction buffer was added to the exonuclease/Pibrentasvir mixture and incubated at $37^{\circ} \mathrm{C}$ for $15 \mathrm{~min}$. The final concentrations of reagents in the $20 \mu \mathrm{L}$ reactions were $50 \mathrm{nM}$ nsp14/nsp10, $500 \mathrm{nM}$ RNA, $0-20 \mu \mathrm{M}$ Pibrentasvir and 5\% DMSO. The $1 \times$ exonuclease reaction buffer contains the following reagents: $40 \mathrm{mM}$ Tris- $\mathrm{HCl} \mathrm{pH} \mathrm{8,} 1.5 \mathrm{mM} \mathrm{MgCl}_{2}$ and $5 \mathrm{mM}$ DTT. After incubation for $15 \mathrm{~min}$, each reaction was quenched by adding $2.2 \mu \mathrm{L}$ of an aqueous solution of EDTA (100 mM). Following desalting using an Oligo Clean \& Concentrator (Zymo Research), the samples were subjected to MALDI-TOF MS (Bruker ultrafleXtreme) analysis. SARS-CoV-2 exonuclease reactions in the presence of Ombitasvir, Daclatasvir, Ritonavir and Lopinavir were conducted analogously to the experiments with Pibrentasvir.

\section{Comparison of SARS-CoV-2 exonuclease reaction for Tenofovir terminated RNA with natural RNA.}

The A-terminated RNA $(2 \mu \mathrm{M})$ and Tenofovir extended RNA $(2 \mu \mathrm{M})$ (sequences shown in Fig. S-4) were annealed by heating to $75^{\circ} \mathrm{C}$ for $3 \mathrm{~min}$ and cooling to room temperature in $1 \times$ exonuclease reaction buffer. After annealing, the two RNAs were mixed in equal volumes. To a $10 \mu \mathrm{L}$ solution of $100 \mathrm{nM}$ exonuclease complex (nsp14/nsp10) in $1 \times$ exonuclease reaction buffer $10 \mu \mathrm{L}$ annealed RNA mixture $\left(1 \mu \mathrm{M}\right.$ each) was added and incubated at $37{ }^{\circ} \mathrm{C}$ for $15 \mathrm{~min}$. The final concentrations of reagents in the $20 \mu \mathrm{L}$ reactions were $50 \mathrm{nM}$ nsp14/nsp10, $\sim 500 \mathrm{nM}$ A-terminated RNA and $\sim 500$ $\mathrm{nM}$ Tenofovir terminated RNA. After incubation for $15 \mathrm{~min}$, each reaction was quenched by adding $2.2 \mu \mathrm{L}$ of an aqueous solution of EDTA $(100 \mathrm{mM})$. Following desalting using an Oligo Clean \& Concentrator (Zymo Research), the samples were subjected to MALDI-TOF MS (Bruker ultrafleXtreme) analysis. 


\section{Molecular docking procedure.}

The chemical structures for Pibrentasvir, Ombitasvir, Elbasvir, Daclatasvir, Ledipasvir and Velpatasvir (Fig. S-1) were obtained from PubChem ${ }^{49}$ and minimized in terms of energy by Density Functional Theory (DFT), with the Becke-3-Lee Yang Parr (B3LYP) method and the standard 6$31 \mathrm{G}^{*}$ basis set, available in Spartan'18 software (Wavefunction, Inc., Irvine, USA) ${ }^{50}$. Since the 3D structure for the SARS-CoV-2 nsp-14 exonuclease was not available, a structural model was built via the on-line tool Swiss Model software (University of Basel, Basel, Switzerland) ${ }^{51}$ using the crystallographic structure of SARS-CoV nsp-14 as template (Protein Data Bank (PDB) code: 5C8T) ${ }^{52}$ and the following SARS-CoV-2 nsp-14 amino acid sequence:

AENVTGLFKDCSKVITGLHPTQAPTHLSVDTKFKTEGLCVDIPGIPKDMTYRRLISMMGFKM NYQVNGYPNMFITREEAIRHVRAWIGFDVEGCHATREAVGTNLPLQLGFSTGVNLVAVPTG YVDTPNNTDFSRVSAKPPPGDQFKHLIPLMYKGLPWNVVRIKIVQMLSDTLKNLSDRVVFV LWAHGFELTSMKYFVKIGPERTCCLCDRRATCFSTASDTYACWHHSIGFDYVYNPFMIDVQ QWGFTGNLQSNHDLYCQVHGNAHVASCDAIMTRCLAVHECFVKRVDWTIEYPIIGDELKIN AACRKVQHMVVKAALLADKFPVLHDIGNPKAIKCVPQADVEWKFYDAQPCSDKAYKIEEL FYSYATHSDKFTDGVCLFWNCNVDRYPANSIVCRFDTRVLSNLNLPGCDGGSLYVNKHAF HTPAFDKSAFVNLKQLPFFYYSDSPCESHGKQVVSDIDYVPLKSATCITRCNLGGAVCRHHA NEYRLYLDAYNMMISAGFSLWVYKQFDTYNLWNTFTRLQ ${ }^{53}$. About five models were generated based on the standard 'auto model' routine of Swiss Model software. The best modeled structure was chosen according to the Qualitative Model Energy Analysis (QMEAN) and Global Model Quality Estimation (GMQE) values.

The molecular docking calculations were performed using GOLD 2020.2 software (Cambridge Crystallographic Data Centre, Cambridge, UK) ${ }^{54}$. Hydrogen atoms were added to the protein structure according to the data inferred by software on the ionization and tautomeric states. Since there are no crystallographic structures for drugs associated with SARS-CoV-2 nsp14 exonuclease, the standard function ChemPLP was used for the molecular docking calculations, selecting an 8 and $10 \AA$ radius spherical cavity around the active binding site. The figures for the docking poses of the largest docking score value was generated with PyMOL Delano Scientific LLC software (Schrödinger, New York, USA) ${ }^{55}$.

\section{Cells and Virus.}

African green monkey kidney cells (Vero, subtype E6) and the human lung epithelial cell line (Calu3 cells) were cultured in high glucose DMEM and low glucose DMEM medium, both complemented with $10 \% \mathrm{FBS}, 100 \mathrm{U} / \mathrm{mL}$ penicillin and $100 \mu \mathrm{g} / \mathrm{mL}$ streptomycin at $37{ }^{\circ} \mathrm{C}$ in a humidified atmosphere with 5\% $\mathrm{CO}_{2}$. SARS-CoV-2 was prepared in Vero E6 cells at a MOI of 0.01. Originally, the isolate was obtained from a nasopharyngeal swab from a confirmed case in Rio de Janeiro, Brazil (GenBank \#MT710714; Institutional Review Broad approval, 30650420.4.1001.0008). All procedures related to virus culture were handled in a Biosafety Level 3 (BSL3) multiuser facility according to WHO guidelines. Virus titers were determined as plaque forming units (PFU)/mL. Virus stocks were kept in $-80^{\circ} \mathrm{C}$ freezers.

\section{Cytotoxicity assay.}

Monolayers of $1.5 \times 10^{4}$ cells in 96-well plates were treated for 3 days with various concentrations (semi-log dilutions from 1000 to $10 \mu \mathrm{M}$ ) of the antiviral drugs. Then, $5 \mathrm{mg} / \mathrm{ml}$ 2,3-bis-(2-methoxy4-nitro-5-sulfophenyl)-2H-tetrazolium-5-carboxanilide (XTT) in DMEM was added to the cells in 
the presence of $0.01 \% \mathrm{~N}$-methyl dibenzopyrazine methyl sulfate (PMS). After incubating for $4 \mathrm{~h}$ at $37{ }^{\circ} \mathrm{C}$, the cells were measured in a spectrophotometer at $492 \mathrm{~nm}$ and $620 \mathrm{~nm}$. The $50 \%$ cytotoxic concentration $\left(\mathrm{CC}_{50}\right)$ was calculated by a non-linear regression analysis of the dose response curves.

\section{Yield-reduction assay.}

Calu-3 cells $\left(5 \times 10^{5}\right.$ cells/well $)$ in 48 -well plates were infected at a MOI of 0.1 for $1 \mathrm{~h}$ at $37^{\circ} \mathrm{C}$. The cells were washed, and various concentrations of compounds were added to DMEM with 10\% FBS. 48-72 h supernatants were collected and the harvested virus was quantified as PFU/mL by titering in Vero E6 cells. A variable slope non-linear regression analysis of the dose response curves was performed to calculate the concentration at which each drug inhibited the virus production by $50 \%$ $\left(\mathrm{EC}_{50}\right), 90 \%\left(\mathrm{EC}_{90}\right)$ and $99 \%\left(\mathrm{EC}_{99}\right)$.

\section{Virus titration.}

Monolayers of Vero E6 cells $\left(2 \times 10^{4}\right.$ cells/well) in 96-well plates were infected with serial dilutions of supernatants containing SARS-CoV-2 for $1 \mathrm{~h}$ at $37{ }^{\circ} \mathrm{C}$. Fresh semi-solid medium containing 2.4\% CMC was added and the culture was maintained for $72 \mathrm{~h}$ at $37{ }^{\circ} \mathrm{C}$. Cells were fixed with $10 \%$ formalin for $2 \mathrm{~h}$ at room temperature and then stained with crystal violet $(0.4 \%)$. Plaque numbers were scored in at least 3 replicates per dilution by independent readers, who were blinded with respect to the source of the supernatant. The virus titers were determined as PFU/mL.

\section{Statistical analysis.}

The assays were performed blinded by one professional, codified and then read by another professional. All experiments were carried out at least three independent times, including a minimum of two technical replicates in each assay. The dose-response curves used to calculate pharmacological parameters were generated by variable slope plot from Prism GraphPad software 8.0. The equations to fit the best curve were generated based on $R^{2}$ values $\geq 0.9$. 


\section{References}

1. Zhu, N. et al. A novel coronavirus from patients with pneumonia in China, 2019. N. Eng. J. Med. 382, 727-733 (2020).

2. V'kovski, P. et al. Coronavirus biology and replication: implications for SARS-CoV-2. Nat. Rev. Microbiol. 19, 155-170 (2021).

3. Shannon, A. et al. Remdesivir and SARS-CoV-2: Structural requirements at both nsp12 RdRp and nsp14 exonuclease active-sites. Antivir. Res. 178, 104793 (2020).

4. Kirchdoerfer, R. N. \& Ward, A. B. Structure of the SARS-CoV nsp12 polymerase bound to nsp7 and nsp8 co-factors. Nat. Commun. 10, 2342 (2019).

5. Gao, Y. et al. Structure of the RNA-dependent RNA polymerase from COVID-19 virus. Science 368, 779-782 (2020).

6. Hillen, H. S. et al. Structure of replicating SARS-CoV-2 polymerase. Nature 584, 154-156 (2020).

7. Bouvet, M. et al. RNA 3'-end mismatch excision by the severe acute respiratory syndrome coronavirus nonstructural protein nsp10/nsp14 exoribonuclease complex. Proc. Natl. Acad. Sci. USA 109, 9372-9377 (2012).

8. Ju, J. et al. Nucleotide analogues as inhibitors of viral polymerases. bioRXiv (2020). https://doi.org/10.1101/2020.01.30.927574

9. Ju, J. et al. Nucleotide analogues as inhibitors of SARS-CoV polymerase. Pharmacol. Res. Perspect. 8, e00674 (2020).

10. Jockusch, S. et al. A library of nucleotide analogues terminate RNA synthesis catalyzed by polymerases of coronaviruses that cause SARS and COVID-19. Antiviral Res. 180, 104857 (2020).

11. Chien, M. et al. Nucleotide analogues as inhibitors of SARS-CoV-2 polymerase, a key drug target for COVID-19. J. Proteome Res. 19, 4690-4697 (2020).

12. Sadeghi, A. et al. Sofosbuvir and daclatasvir compared with standard of care in the treatment of patients admitted to hospital with moderate or severe coronavirus infection (COVID-19): a randomized controlled trial. J. Antimicrob. Chemother. 75, 3379-3385 (2020).

13. Vicenti, I., Zazzi, M. \& Saladini, F. SARS-CoV-2 RNA-dependent RNA polymerase as a therapeutic target for COVID-19. Expert Opin. Ther. Pat. 31, 325-337 (2021).

14. Gordon, C. J. et al. Remdesivir is a direct-acting antiviral that inhibits RNA-dependent RNA polymerase from severe acute respiratory syndrome coronavirus 2 with high potency. J. Biol. Chem. 295, 6785-6797 (2020). 
15. Bravo, J. P. K., Dangerfield, T. L., Taylor, D. W. \& Johnson, K. A. Remdesivir is a delayed translocation inhibitor of SARS-CoV-2 replication. Molec. Cell 81, 1548-1552 (2021).

16. Jockusch, S. et al. Sofosbuvir terminated RNA is more resistant to SARS-CoV-2 proofreader than RNA terminated by Remdesivir. Sci. Rep. 10, 16577 (2020).

17. Khater, S., Dasgupta, N. \& Das, G. Combining SARS-CoV-2 proofreading exonuclease and RNA-dependent RNA polymerase inhibitors as a strategy to combat COVID-19: a high-throughput in silico screen. OSF Preprints. (2020). https://osf.io/7x 5ek/

18. Smith, M. A., Regal, R. E. \& Mohammad, R. A. Daclatasvir: A NS5A replication complex inhibitor for hepatitis C infection. Ann. Pharmacother. 50, 39-46 (2016).

19. Gordon, D. E. et al. A SARS-CoV-2 protein- interaction map reveals targets for drug repurposing. Nature 583, 459-468 (2020).

20. Sacramento, C. et al. In vitro antiviral activity of the anti-HCV drugs daclatasvir and sofosbuvir against SARS-CoV-2, the aetiological agent of COVID-19. J. Antimicrob. Chemother. (2021). https://doi.org/10.1093/jac/dkab072

21. Sanders, R. Can hepatitis C drugs help remdesivir fight COVID-19?

https://news.berkeley.edu/2021/02/03/can-hepatitis-c-drugs-help-remdesiver-fight-covid-19/

22. Nguyenla, X. et al. Discovery of SARS-CoV-2 antiviral synergy between remdesivir and approved drugs in human lung cells. bioRxiv (2020). https://doi.org/10.1101/2020.09.18.302398

23. Shannon, A. et al. Rapid incorporation of favipiravir by the fast and permissive viral RNA polymerase complex results in SARS-CoV-2 lethal mutagenesis. Nature Comm. 11, 4682 (2020).

24. Sheahan, T. R. et al. An orally bioavailable broad-spectrum antiviral inhibits SARS-CoV-2 in human airway epithelial cell cultures and multiple coronaviruses in mice. Sci. Transl. Med. 12, eabb5883. (2020).

25. Good, S. S. et al. AT-527, a double prodrug of a guanosine nucleotide analog, is a potent inhibitor of SARS-CoV-2 in vitro and a promising oral antiviral for treatment of COVID-19. Antimicrob. Agents Chemother. 65, e02479-20 (2021).

26. $\mathrm{Chu}, \mathrm{H}$. et al. Comparative replication and immune activation profiles of SARS-CoV-2 and SARS-CoV in human lungs: an ex vivo study with implications for the pathogenesis of COVID-19. Clin. Infect. Dis. 71, 1400-1409 (2020).

27. Chu, H. et al. Comparative tropism, replication kinetics, and cell damage profiling of SARSCoV-2 and SARS-CoV with implications for clinical manifestations, transmissibility, and laboratory studies of COVID-19: an observational study. Lancet Microbe 1, e14-23 (2020).

28. https://www.cdc.gov.tw/File/Get/ht8jUiB_MI-aKnlwstwzvw 
29. Ju, J. et al. Therapeutics for COVID-19. PCT patent application. Date of filing: Jan. 29, 2021.

30. Narayanan, N. \& Nair, D. T. Ritonavir may inhibit exoribonuclease activity of nsp14 from the SARS-CoV-2 virus and potentiate the activity of chain terminating drugs. Int. J. Biol. Macromol. 168, 272-278 (2021).

31. Cao, B. et al. A trial of Lopinavir-Ritonavir in adults hospitalized with severe Covid-19. N. Engl. J. Med. 382, 1787-1799 (2020).

32. Essam, A. Comparison of remdesivir versus lopinavir/ritonavir and remdesivir combination in COVID-19 patients. https://clinicaltrials.gov/ct2/show/NCT04738045

33. Blasiak, A. et al. IDentif.AI: Rapidly optimizing combination therapy design against severe Acute Respiratory Syndrome Coronavirus 2 (SARS-CoV-2) with digital drug development. Bioeng. Transl. Med. 6, e10196 (2020).

34. https://pdf.hres.ca/dpd_pm/00040336.PDF

35. Humeniuk, R. Safety, tolerability, and pharmacokinetics of Remdesivir, an antiviral for treatment of COVID-19, in healthy subjects. Clin. Translat. Sci. 13, 896-906 (2020).

36. Gribble, J. et al. The coronavirus proofreading exoribonuclease mediates extensive viral recombination. PLoS Pathog. 17, e1009226 (2021).

37. Baddock, H.T. et al. Characterisation of the SARS-CoV-2 ExoN (nsp14 ${ }^{\text {ExoN }}$-nsp10) complex: implications for its role in viral genome stability and inhibitor identification. bioRxiv (2020) https://doi.org/10.1101/2020.08.13.248211

38. Chen, T. et al. Synergistic inhibition of SARS-CoV-2 replication using Disulfiram/Ebselen and Remdesivir. ACS Pharmacol. Transl. Sci. 4, 898-907 (2021).

39. Bafna, K. et al. Hepatitis $\mathrm{C}$ virus drugs that inhibit the SARS-CoV-2 papain-like protease synergize with Remdesivir to suppress viral replication in cell culture. Cell Rep. 35, 109133 (2021).

40. Driouich, J. S. et al. Favipiravir antiviral efficacy against SARS-CoV-2 in a hamster model. Nature Comm. 12, 1735 (2021).

41. Agrawal, U., Raju, R. \& Udwadia, Z. F. Favipiravir: A new and emerging antiviral option in COVID-19. Med. J. Armed Forces India 76, 370-376 (2020).

42. Furuta, Y. et al. Favipiravir (T-705), a novel viral polymerase inhibitor. Antivir. Res. 100, 446454 (2013).

43. Furuta, Y. et al. Favipiravir (T-705), a broad-spectrum inhibitor of viral RNA polymerase. Proc. Jpn. Acad. Ser. B Phys. Biol. Sci. 93, 449-463 (2017). 
44. Naydenova, K. et al. Structure of the SARS-CoV-2 RNA-dependent RNA polymerase in the presence of favipiravir-RTP. Proc. Natl. Acad. Sci. USA 118, e2021946118 (2021).

45. Peng, Q. et al. Structural basis of SARS-CoV-2 polymerase inhibition by Favipiravir. Innovation N.Y. 2, 100080 (2021).

46. Efficacy and safety of molnupiravir (MK-4482) in hospitalized adult participants with COVID19 (MK-4482-001). First post Oct. 5, 2020. Last post May 7, 2021. https://www.clinicaltrials.gov/ct2/show/NCT04575584

47. Shannon, A. et al. Protein-primed RNA synthesis in SARS-CoVs and structural basis for inhibition by AT-527. bioRxiv. https://doi.org/10.1101/2021.03.23.436564 (2021).

48. Blanco-Melo, D. et al. Imbalanced host response to SARS-CoV-2 drives development of COVID-19. Cell 181, 1036-1045 (2020).

49. https://pubchem.ncbi.nlm.nih.gov/ accessed in February 2021.

50. https://www.wavefun.com/ accessed in February 2021.

51. https://swissmodel.expasy.org/ accessed in February 2021.

52. Ma, Y. et al. Structural basis and functional analysis of the SARS coronavirus nsp14-nsp10 complex. Proc. Natl. Acad. Sci. USA 112, 9436-9441 (2015).

53. https://www.ncbi.nlm.nih.gov/protein/YP_009725309.1?report=fasta accessed in February 2021.

54. https://www.ccdc.cam.ac.uk/solutions/csd-discovery/components/gold/ accessed in February 2021.

55. https:// www.pymol.org/2/ accessed in February 2021 


\section{Acknowledgements}

This work was supported by the Jack Ma Foundation, a gift from Columbia Engineering Member of the Board of Visitors Dr. Bing Zhao, and Fast Grants (to Jingyue Ju), the Maloris Foundation and the Memorial Sloan-Kettering Core Grant (P30CA008748) (to Dinshaw J. Patel), a grant from The JPB Foundation to Rockefeller University (to Thomas Tuschl). Funding was also provided by Conselho Nacional de Desenvolvimento Científico e Tecnológico (CNPq), Fundação de Amparo à Pesquisa do Estado do Rio de Janeiro (FAPERJ) and Coordenação de Aperfeiçoamento de Pessoal de Nível Superior - Brasil (CAPES) - Finance Code 001 (to Thiago Moreno L. Souza and Patricia T. Bozza). CNPq, CAPES and FAPERJ also support the National Institutes of Science and Technology Program (INCT-IDPN). Oswaldo Cruz Foundation/FIOCRUZ supports this study under the auspices of the Inova Program (B3-Bovespa funding) (to Thiago Moreno L. Souza). Dr. Andre Sampaio from Farmanguinhos, platform RPT11M, is acknowledged for kindly donating the Calu-3 cells. We thank Dr. Andrew Owen from the University of Liverpool for insightful discussions.

\section{Author contributions}

X.W., C.Q.S., S.J., O.A.C, C.T., N. F.-R., M.C., J.R.T., W.X., C.M. and A.G. performed the experiments. X.W., C.Q.S., S.J., C.T., N.F.-R., M.C., J.R.T., P.T.B., X.L., S.K., J.J.R., T.M.L.S. and J.J. analyzed the data. X.W., C.Q.S., S.J., C.T., M.C., J.R.T., X.L., S.K., P.T.B., J.J.R., T.M.L.S. and J.J. prepared the manuscript. C.Q.S., D.J.P., T.T., T.M.L.S. and J.J. conceptualized the experiments.

\section{Competing interests}

The authors declare no competing interests.

\section{Additional information}

Correspondence and requests for materials should be addressed to J.J. and T.M.L.S. 


\section{SUPPLEMENTARY INFORMATION}

\section{Combination of Antiviral Drugs to Inhibit SARS-CoV-2 Polymerase and Exonuclease as Potential COVID-19 Therapeutics}

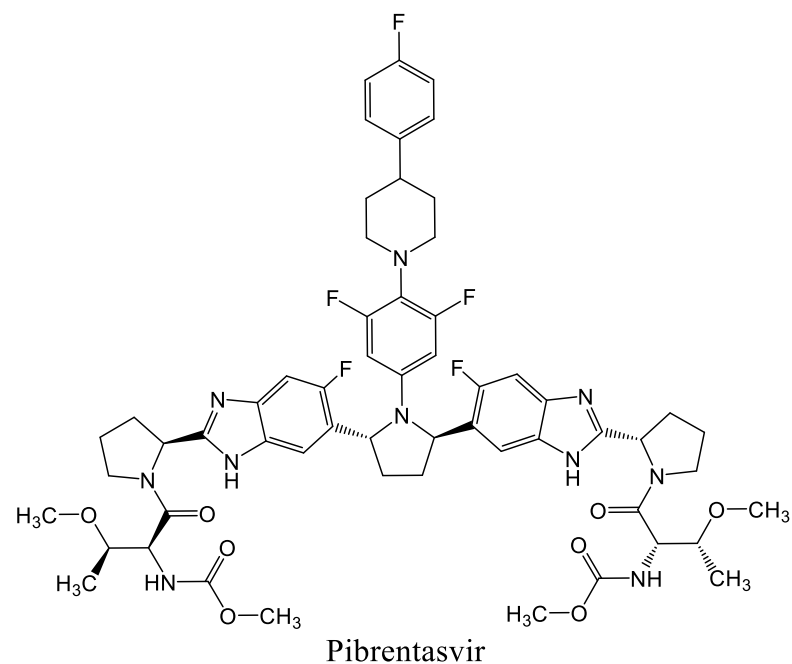

Pibrentasvir

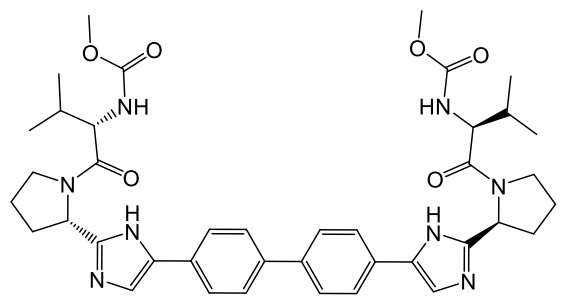

Daclatasvir

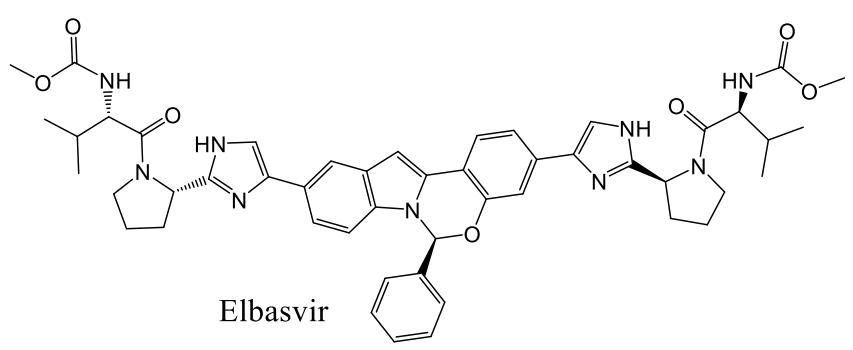

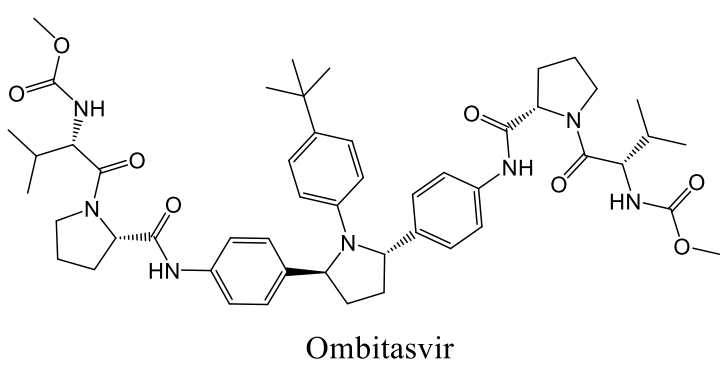

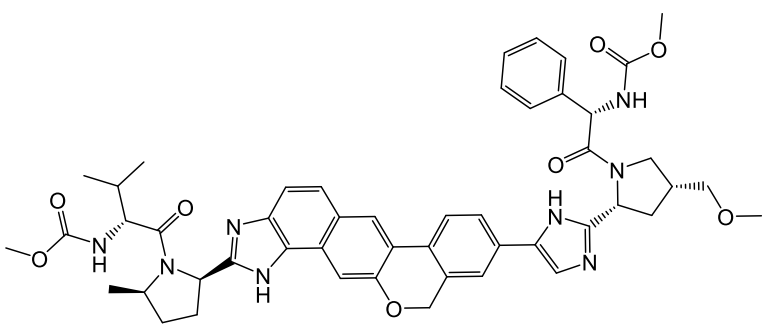

Velpatasvir

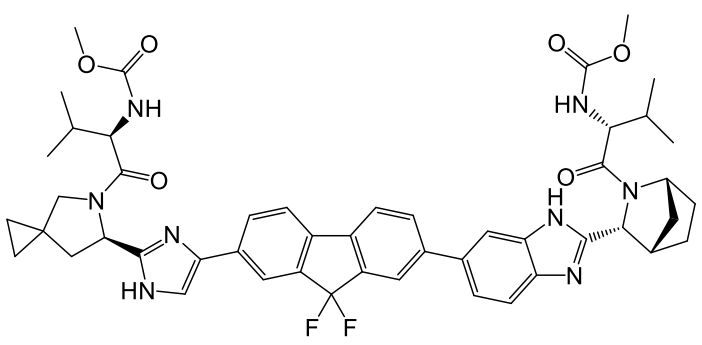

Ledipasvir

Fig. S-1 Structures of HCV NS5A inhibitors: Pibrentasvir, Ombitasvir, Daclatasvir, Velpatasvir, Elbasvir and Ledipasvir. 


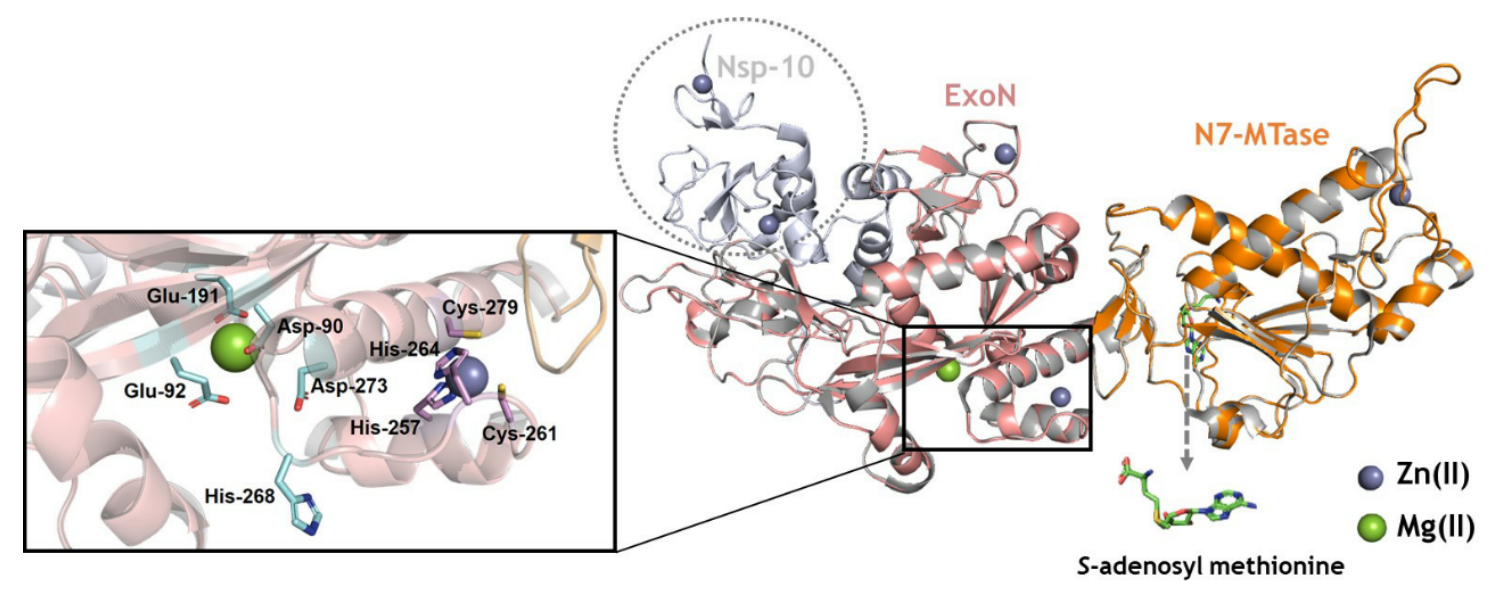

Fig. S-2 Superposition of the SARS-CoV nsp14 (PDB code: 5C8T, in gray) and SARS-CoV-2 nsp14 model (beige and orange for ExoN and N7-MTase domains, respectively). Selected amino acid residues in the exonuclease catalytic site are represented as stick representations in cyan. The co-substrate involved in methyltransferase, $S$-adenosyl methionine (SAM), is represented using a stick model in green. The hydrogen atoms were omitted for better clarity. The oxygen (red), nitrogen (dark blue) and sulfur atoms (yellow) are presented in the stick structure. The $\mathrm{Mg}^{++}$(green) and $\mathrm{Zn}^{++}$ (indigo blue) ions are represented as spheres. 

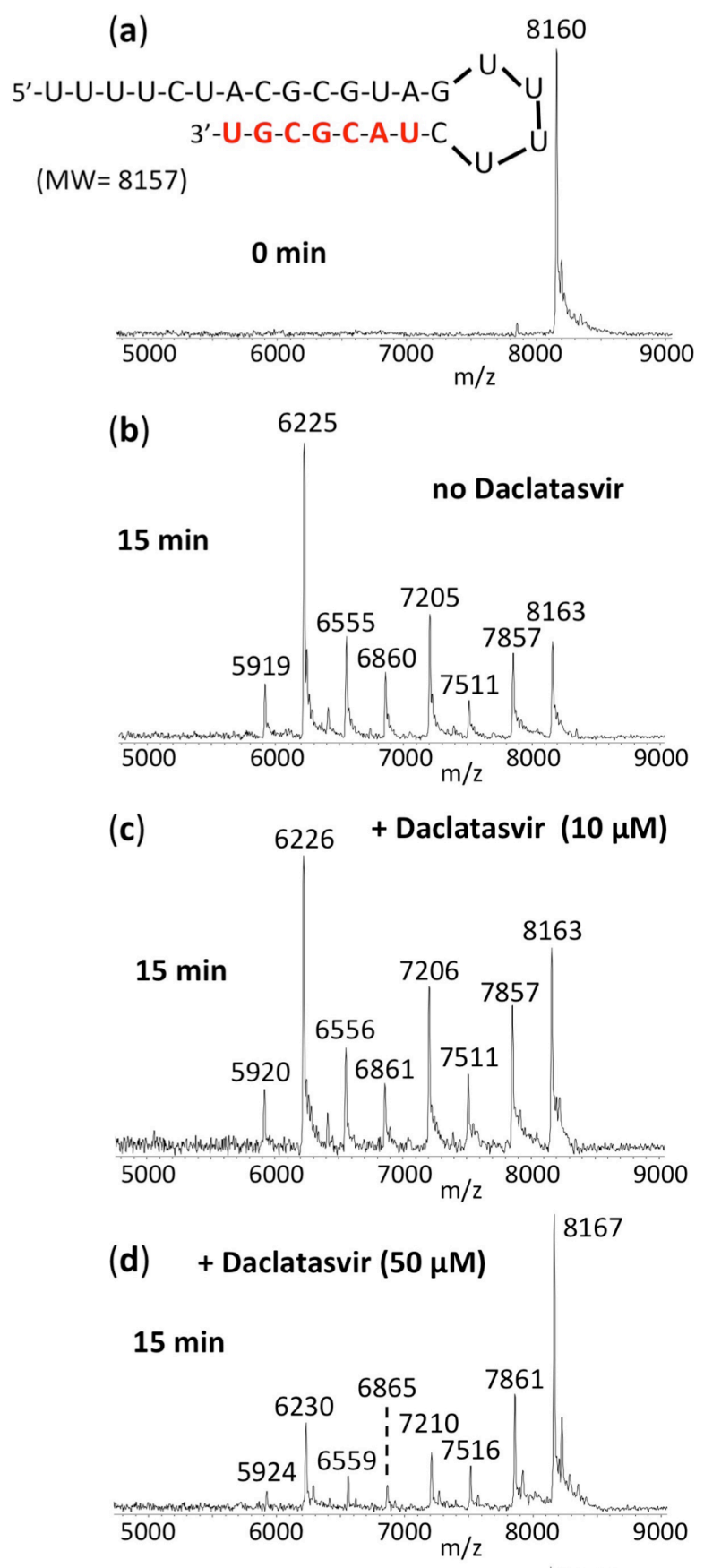

(e) + Daclatasvir $(100 \mu \mathrm{M})$

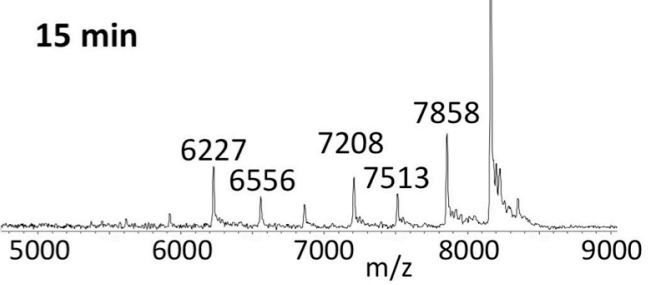

Fig. S-3 Inhibition of SARS-CoV-2 exonuclease activity by Daclatasvir. A mixture of $500 \mathrm{nM}$ RNA (sequence shown at the top of the figure) and $50 \mathrm{nM}$ SARS-CoV-2 pre-assembled exonuclease complex (nsp14/nsp10) was incubated in buffer solution at $37{ }^{\circ} \mathrm{C}$ for $15 \mathrm{~min}$ in the absence (b) and presence of varying amounts of Daclatasvir dihydrochloride (c-e). In this set of experiments, the water-soluble dihydrochloride form of Daclatasvir was used without DMSO. The RNA (a) and the products of the exonuclease reaction (b-e) were analyzed by MALDI-TOF MS. The signal intensity was normalized to the highest peak. The peak at $8160 \mathrm{Da}$ corresponds to the intact RNA (8157 Da expected). In the absence of Daclatasvir, exonuclease activity caused nucleotide cleavage from the 3'-end of the RNA as shown by the 7 lower molecular weight fragments corresponding to cleavage of 1-7 nucleotides (b). With increasing amounts of Daclatasvir, exonuclease activity was reduced as shown by the reduced intensities of the fragmentation peaks and increased intact RNA peak (c-e). 


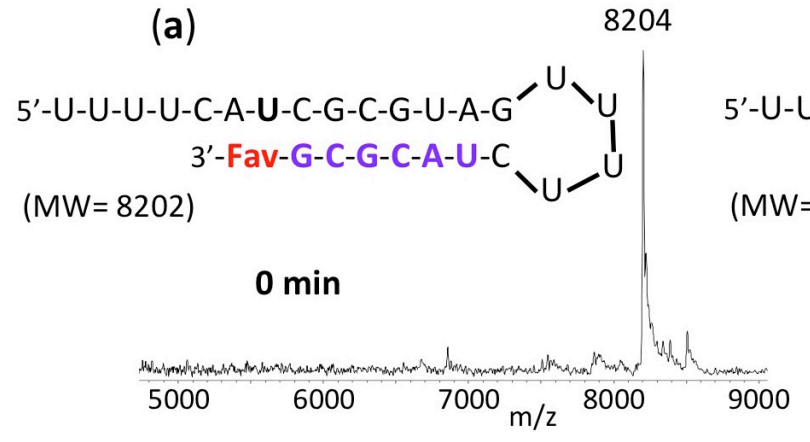

(b)

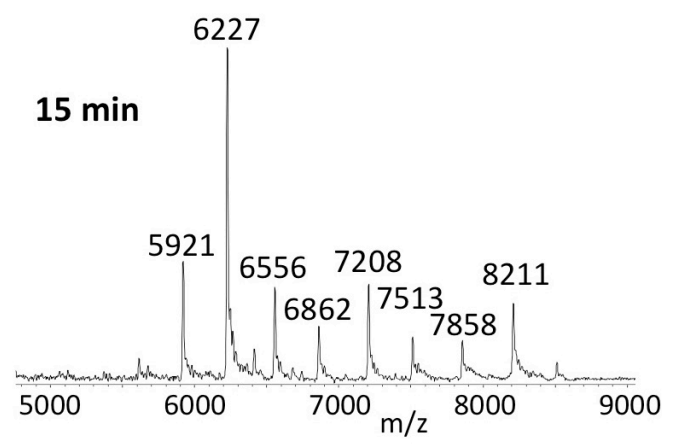

(c) + Pibrentasvir $(20 \mu \mathrm{M})$

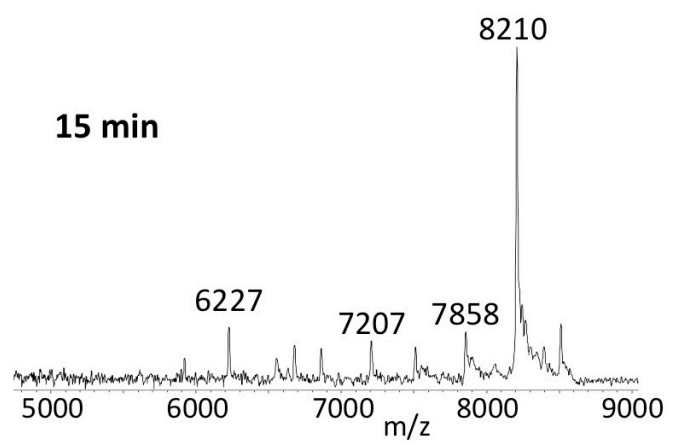

(d) 8204

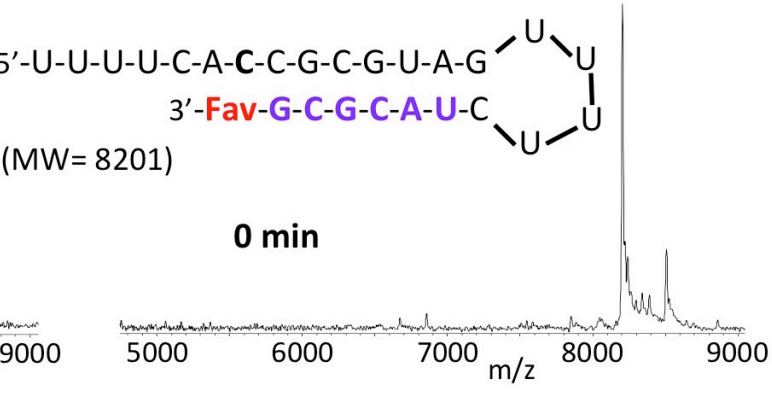

(e)

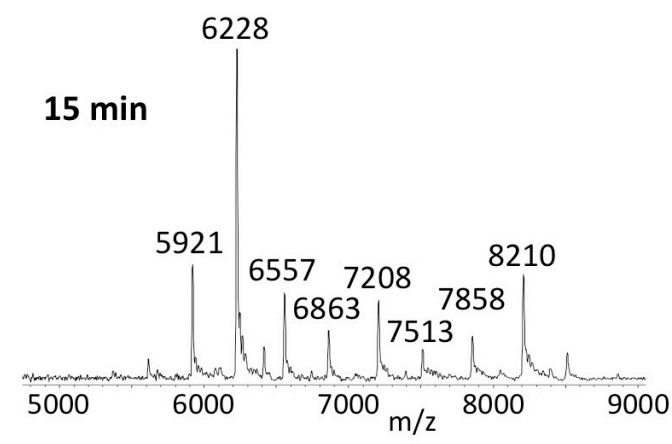

(f) + Pibrentasvir $(20 \mu \mathrm{M})$

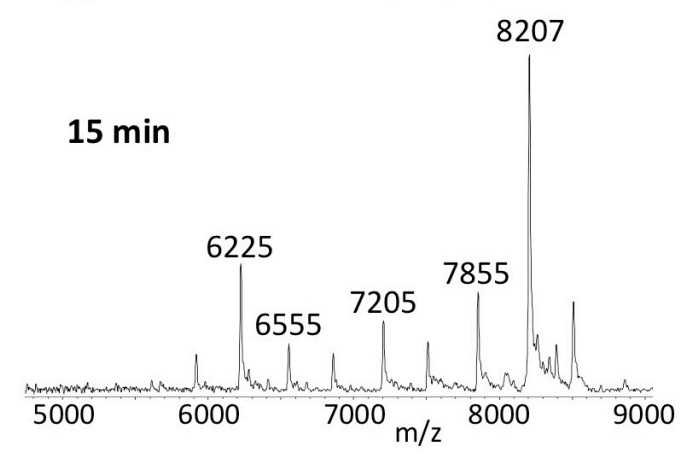

Fig. S-4: Inhibition of SARS-CoV-2 exonuclease activity by Pibrentasvir for Favipiravir (Fav) terminated RNAs. A mixture of $500 \mathrm{nM}$ RNAs (sequences shown at the top of the figure) and 50 nM SARS-CoV-2 pre-assembled exonuclease complex (nsp14/nsp10) were incubated in buffer solution at $37{ }^{\circ} \mathrm{C}$ for $15 \mathrm{~min}$ in the absence (b, e) and presence of $20 \mu \mathrm{M}$ Pibrentasvir (c, f). The intact RNAs (a, d) and the products of the exonuclease reactions (b, c, e, f) were analyzed by MALDI-TOF MS. The signal intensity was normalized to the highest peak. In the absence of Pibrentasvir, exonuclease activity caused nucleotide cleavage from the 3'-end of the RNA as shown by the lower molecular weight fragments corresponding to cleavage of 1-7 nucleotides $(\mathrm{b}, \mathrm{e})$. When $20 \mu \mathrm{M}$ Pibrentasvir was added, exonuclease activity was reduced as shown by the reduced intensities of the fragmentation peaks and increased peak height of the intact RNA (c, f). These results indicate that the SARS-CoV-2 exonuclease activity is substantially inhibited by Pibrentasvir for Favipiravir terminated RNAs. 


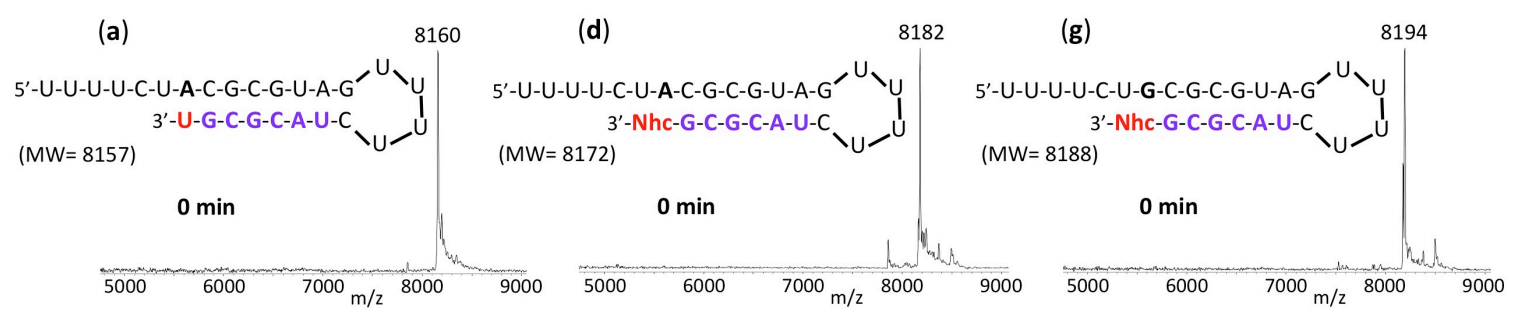

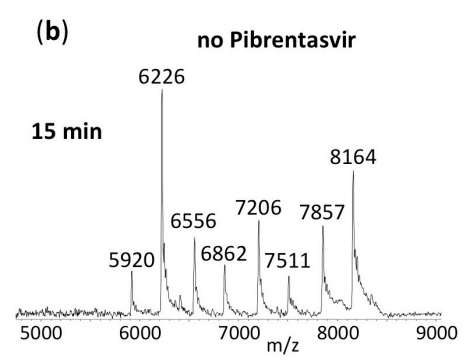

(c) + Pibrentasvir $(20 \mu \mathrm{M})$

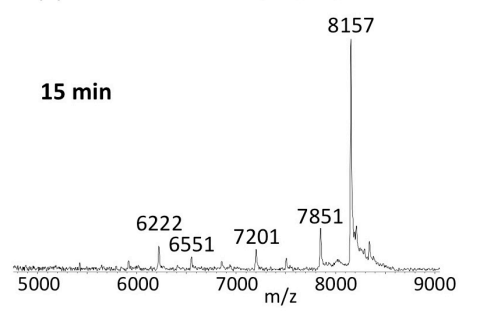

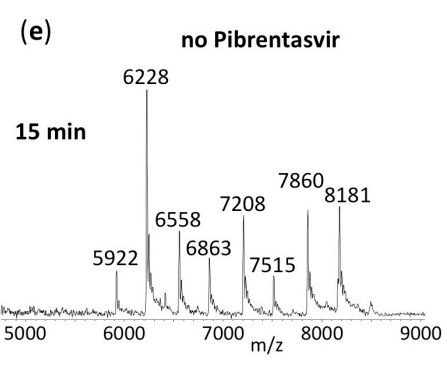

(f) $\quad+$ Pibrentasvir $(20 \mu \mathrm{M})$

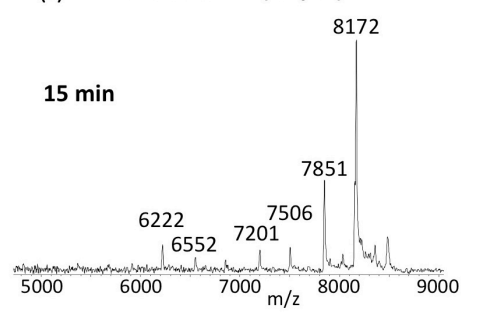

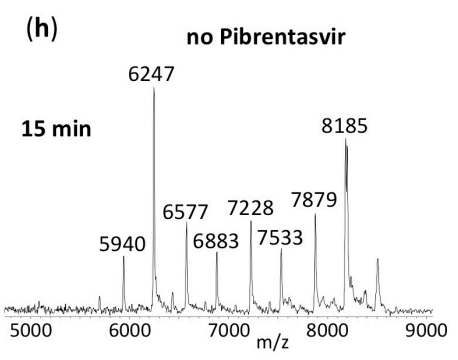

(i) + Pibrentasvir $(20 \mu \mathrm{M})$

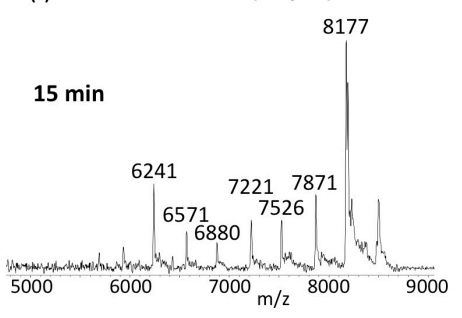

Fig. S-5: Inhibition of SARS-CoV-2 exonuclease activity by Pibrentasvir for natural RNA and $\mathbf{N}^{4}$-hydroxycytidine (Nhc) terminated RNAs. A mixture of $500 \mathrm{nM}$ RNAs (sequences shown at the top of the figure) and $50 \mathrm{nM}$ SARS-CoV-2 pre-assembled exonuclease complex (nsp14/nsp10) were incubated in buffer solution at $37^{\circ} \mathrm{C}$ for $15 \mathrm{~min}$ in the absence (b, e, h) and presence of $20 \mu \mathrm{M}$ Pibrentasvir (c, f, i). The intact RNAs $(\mathrm{a}, \mathrm{d}, \mathrm{g})$ and the products of the exonuclease reactions $(\mathrm{b}, \mathrm{c}, \mathrm{e}$, $\mathrm{f}, \mathrm{h}, \mathrm{i})$ were analyzed by MALDI-TOF MS. The signal intensity was normalized to the highest peak. In the absence of Pibrentasvir, exonuclease activity caused nucleotide cleavage from the 3'-end of the RNA as shown by the lower molecular weight fragments corresponding to cleavage of 1-7 nucleotides (b, e, h). When $20 \mu \mathrm{M}$ Pibrentasvir was added, exonuclease activity was reduced as shown by the reduced intensities of the fragmentation peaks and increased peak height of the intact RNA (c, f, i). These results indicate that the SARS-CoV-2 exonuclease activity is substantially inhibited by Pibrentasvir for NHC terminated RNAs. 


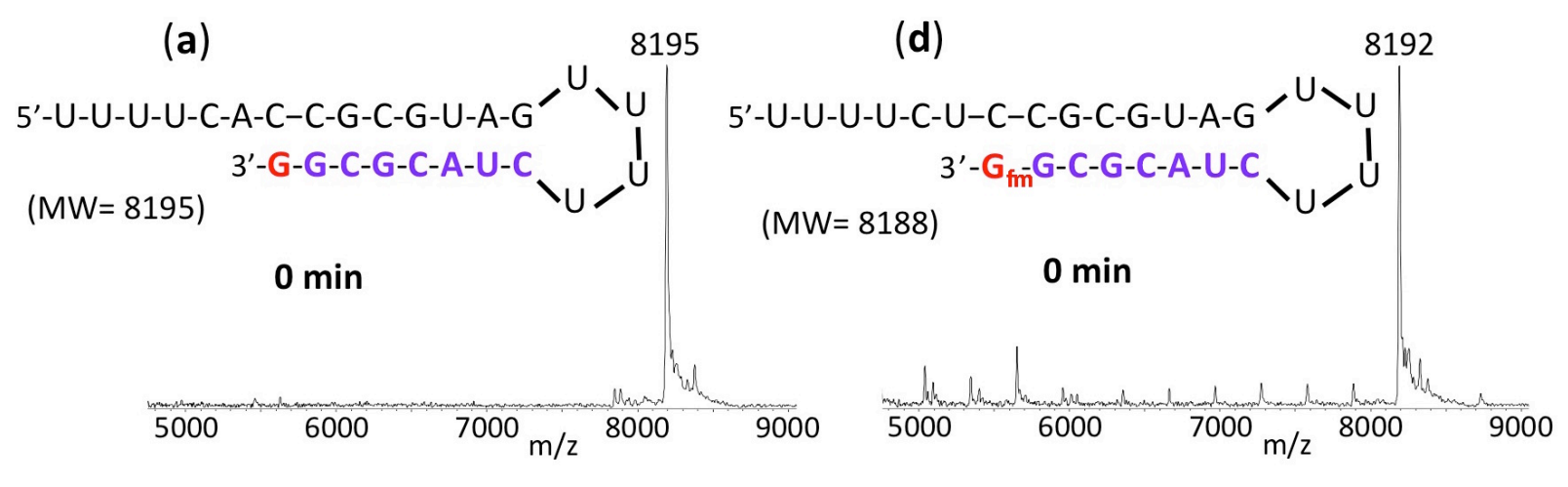

(b)

no Pibrentasvir

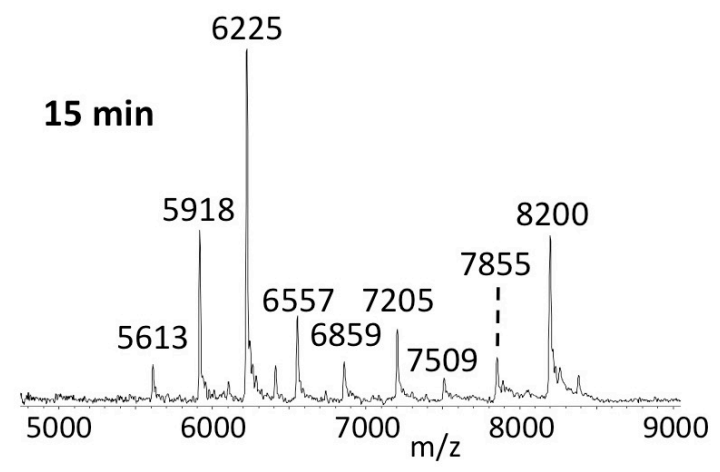

(c) + Pibrentasvir $(20 \mu \mathrm{M})$

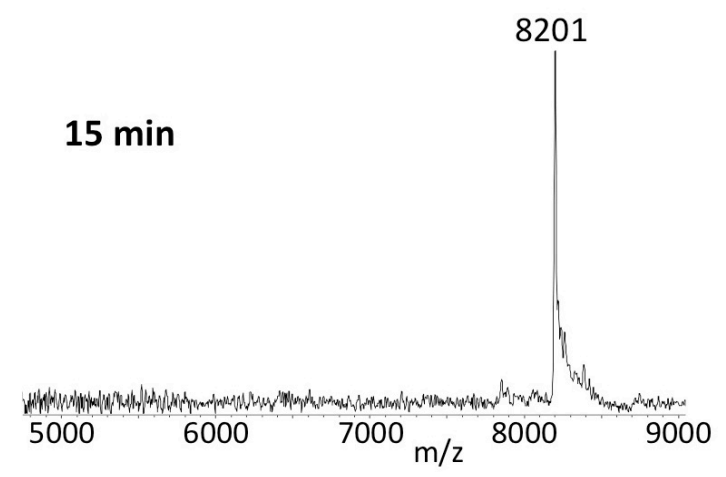

(e)

no Pibrentasvir

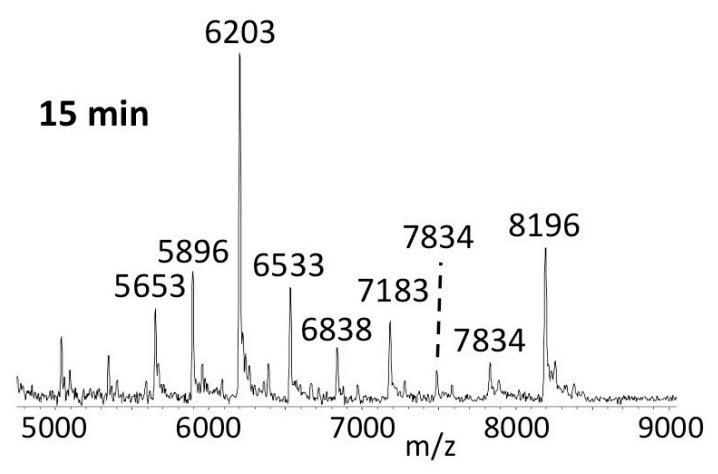

(f) + Pibrentasvir $(20 \mu \mathrm{M})$

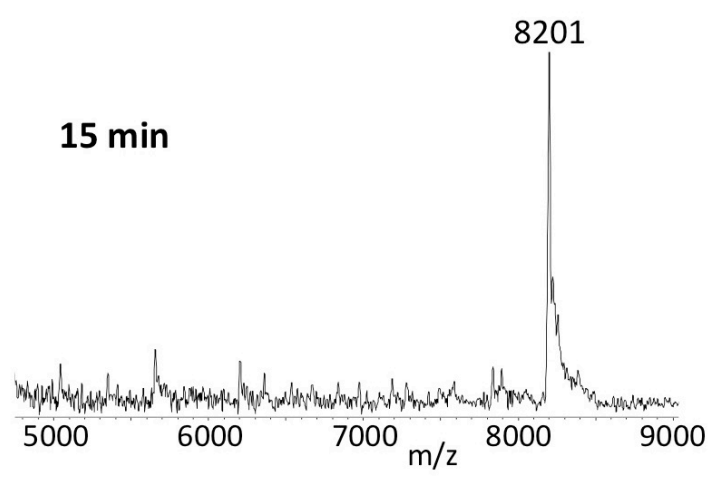

Fig. S-6: Inhibition of SARS-CoV-2 exonuclease activity by Pibrentasvir for natural RNA and AT-9010 $\left(\mathbf{G}_{\mathrm{fm}}\right)$ terminated RNA. A mixture of $500 \mathrm{nM}$ RNAs (sequences shown at the top of the figure) and $50 \mathrm{nM}$ SARS-CoV-2 pre-assembled exonuclease complex (nsp14/nsp10) were incubated in buffer solution at $37^{\circ} \mathrm{C}$ for $15 \mathrm{~min}$ in the absence (b, natural RNA; e, $\mathrm{G}_{\mathrm{fm}}$ terminated RNA) and presence of $20 \mu \mathrm{M}$ Pibrentasvir (c, natural RNA; $\mathrm{f}, \mathrm{G}_{\mathrm{fm}}$ terminated RNA). The intact RNAs (a, d) and the products of the exonuclease reactions (b-f) were analyzed by MALDI-TOF MS. The signal intensity was normalized to the highest peak. In the absence of Pibrentasvir, exonuclease activity caused nucleotide cleavage from the 3'-end of the natural RNA as shown by the lower molecular weight fragments corresponding to cleavage of 1-8 nucleotides (b, e). When $20 \mu \mathrm{M}$ Pibrentasvir was added, exonuclease activity was almost completely abolished as shown by the near absence of the fragmentation peaks and increased peak height of the intact RNA (c, f). These results indicate that the SARS-CoV-2 exonuclease activity is substantially inhibited by Pibrentasvir for both natural and AT-9010 $\left(\mathrm{G}_{\mathrm{fm}}\right)$ terminated RNA. 


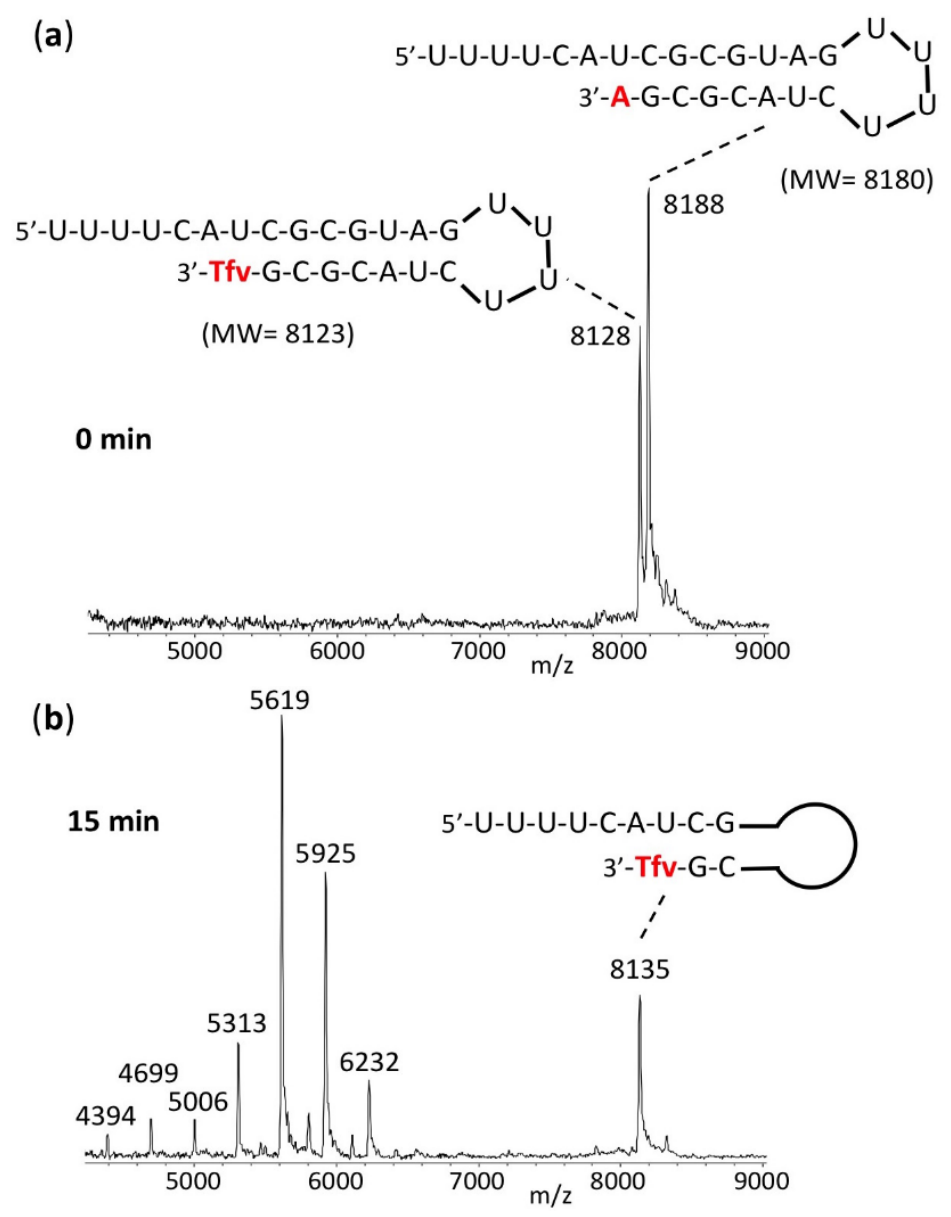

Fig. S-7 SARS-CoV-2 exonuclease activity for a mixture of natural RNA and Tenofovir (Tfv) terminated RNA. A mixture of $\sim 500 \mathrm{nM}$ RNAs (sequences shown at the top of the figure) and 50 nM SARS-CoV-2 pre-assembled exonuclease complex (nsp14/nsp10) were incubated in buffer solution at $37{ }^{\circ} \mathrm{C}$ for $15 \mathrm{~min}(\mathrm{~b})$. The untreated RNA mixture (0 min) (a) and the products of the exonuclease reactions (b) were analyzed by MALDI-TOF MS. Exonuclease activity caused nucleotide cleavage from the 3'-end of the natural RNA as shown by the lower molecular weight fragments corresponding to cleavage of up to 12 nucleotides (b). No detectable MS peak was observed for the full-length natural RNA. However, a large MS peak for intact Tfv terminated RNA was observed at $8135 \mathrm{Da}$ (b) demonstrating that Tfv terminated RNA shows high resistance to SARS-CoV-2 exonuclease activity compared to natural RNA. 
A

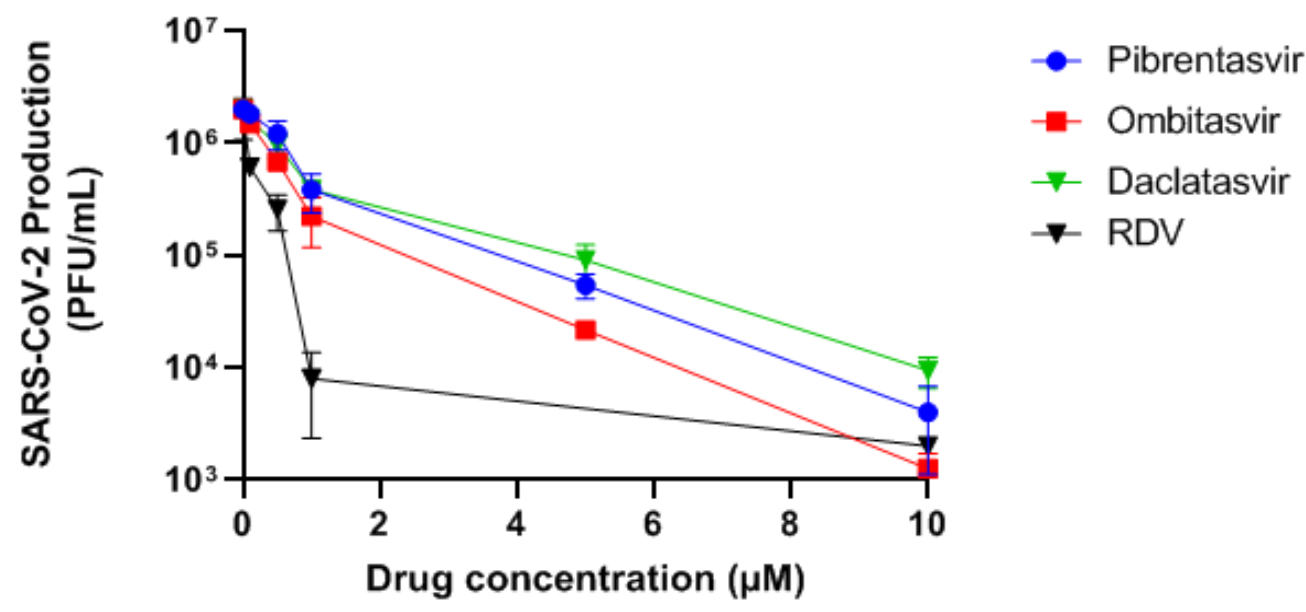

B

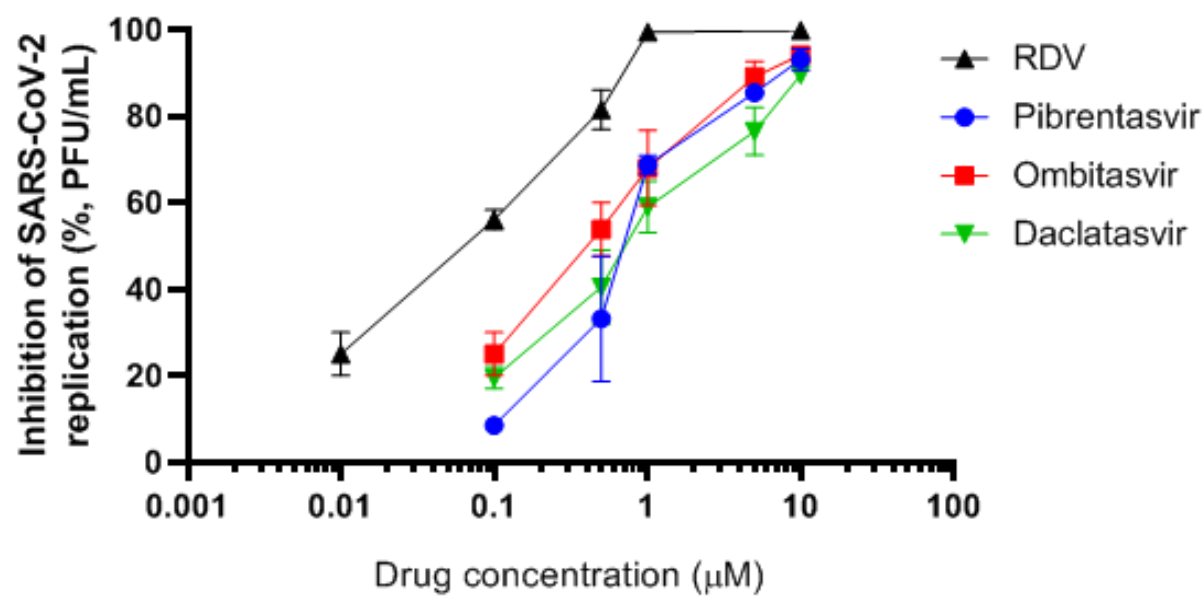

Fig. S-8 Antiviral activity of Pibrentasvir, Ombitasvir and Daclatasvir against SARS-CoV-2. Calu-3 cells, at a density of $5 \times 10^{5}$ cells/well in 48 -well plates, were infected with SARS-CoV-2 at a MOI of 0.1 , for $1 \mathrm{~h}$ at $37{ }^{\circ} \mathrm{C}$. An inoculum was removed and cells were washed and incubated with fresh DMEM containing 2\% FBS and the indicated concentration of the drugs, including Remdesivir (RDV) as the control. Supernatants were assessed after 48-72 $\mathrm{h}$. Viral replication in the culture supernatant was measured as PFU/mL by titering in Vero E6 cells. Results are displayed as virus titers (A) and percentage of inhibition (B). The data represent means \pm SEM of three independent experiments. 


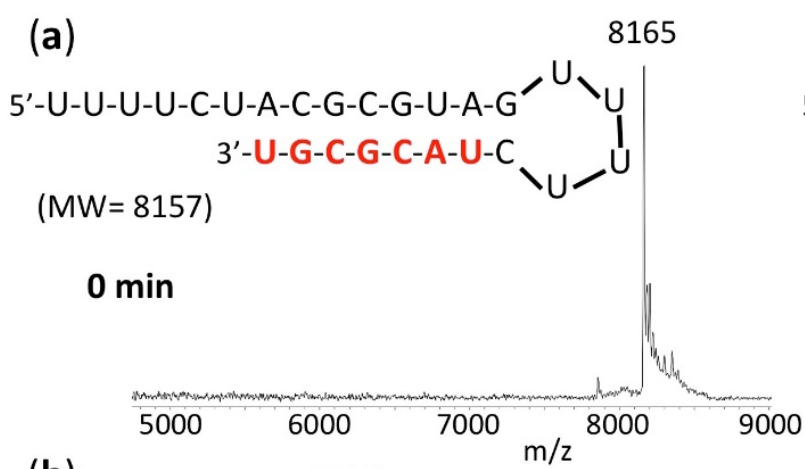

(b)

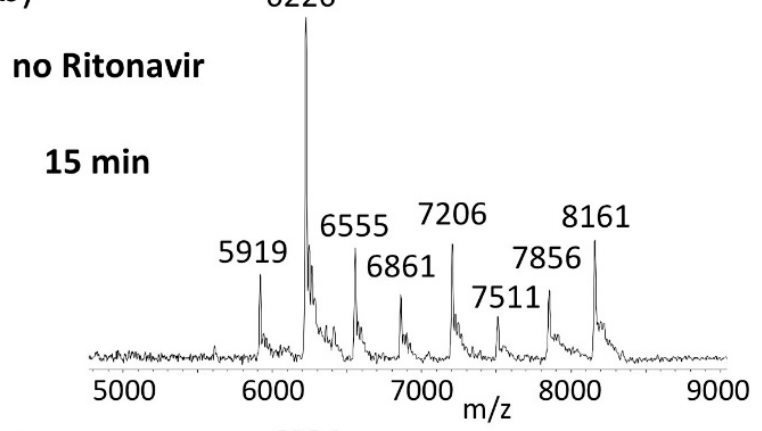

(c)

6224

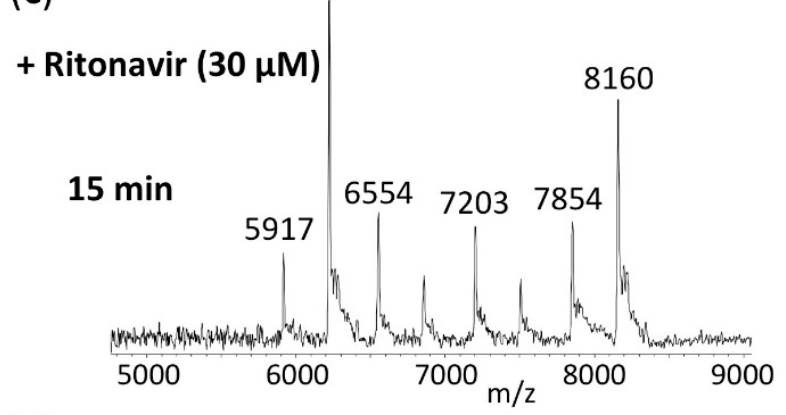

(d)

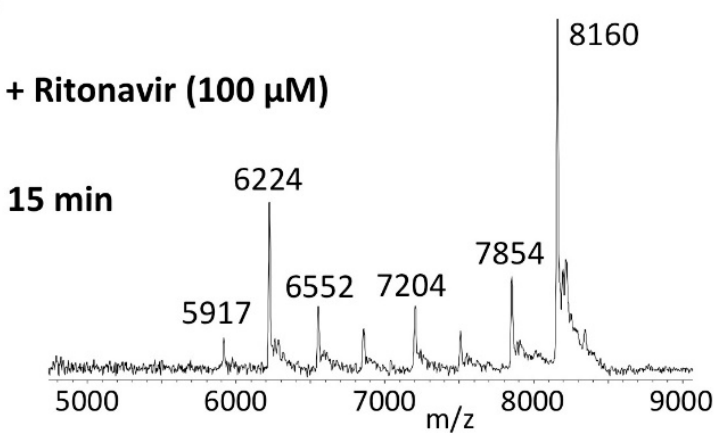

(e)

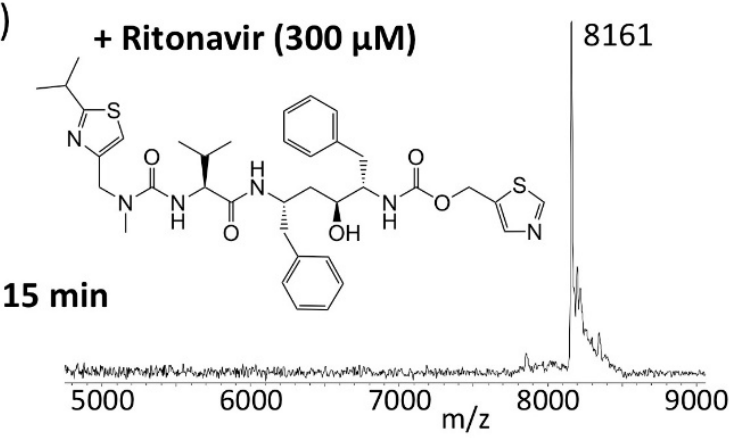

(f)

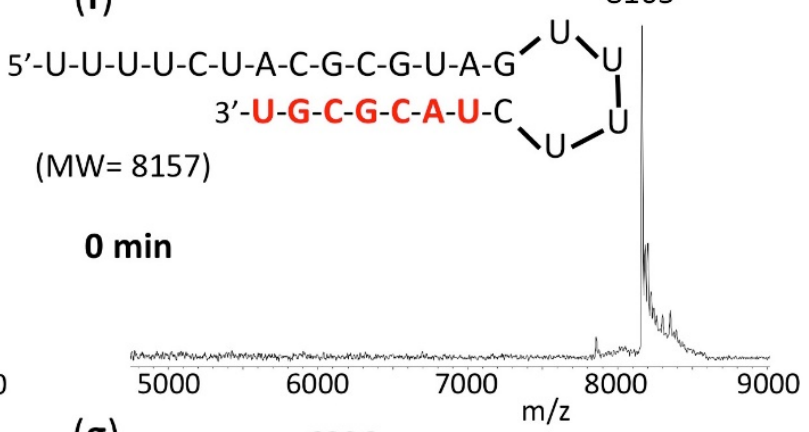

(g)

6226

no Lopinavir

$15 \mathrm{~min}$

(h)
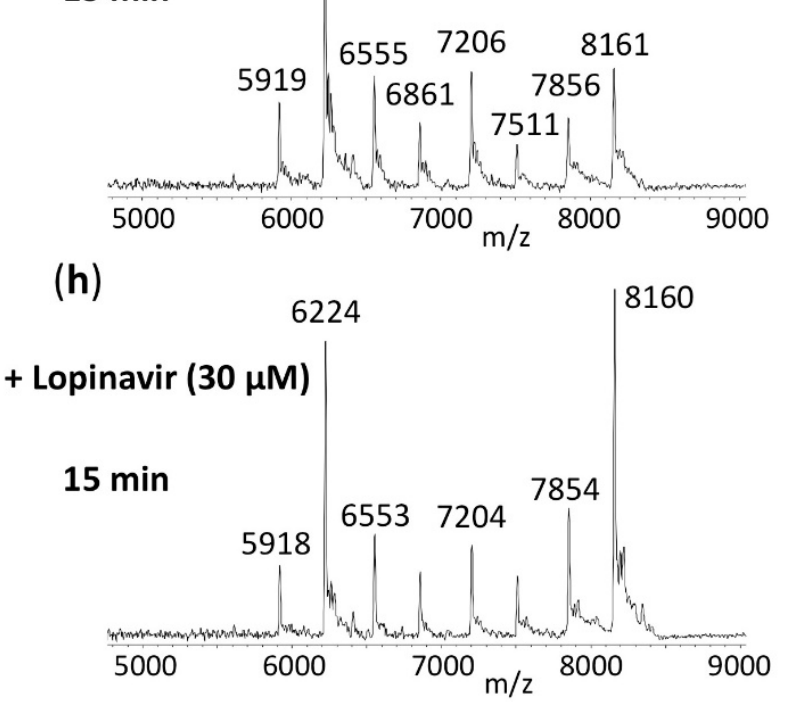

(i)

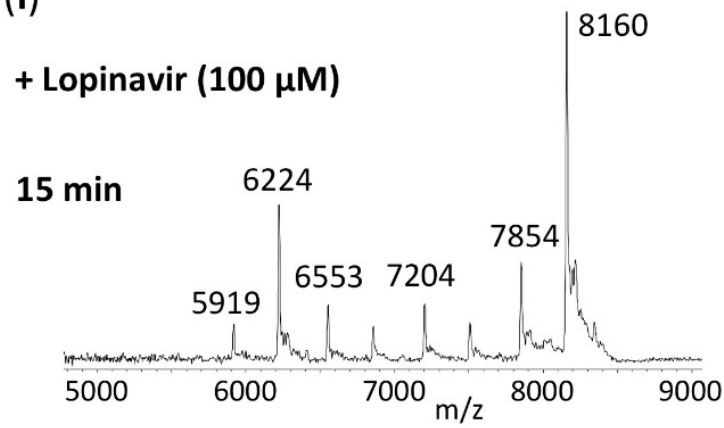

(j)

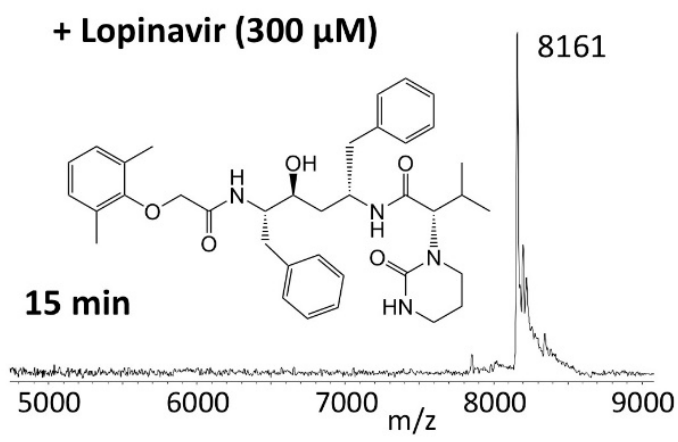


Fig. S-9 Inhibition of SARS-CoV-2 exonuclease activity by Ritonavir and Lopinavir. A mixture of $500 \mathrm{nM}$ RNA (sequence shown at the top of the figure) and $30 \mathrm{nM} \mathrm{SARS-CoV-2}$ pre-assembled exonuclease complex (nsp14/nsp10) was incubated in buffer solution at $37{ }^{\circ} \mathrm{C}$ for $15 \mathrm{~min}$ in the absence $(\mathrm{b}, \mathrm{g})$ and presence of $30 \mu \mathrm{M}(\mathrm{c}, \mathrm{h}), 100 \mu \mathrm{M}(\mathrm{d}, \mathrm{i})$ or $300 \mu \mathrm{M}(\mathrm{e}, \mathrm{j})$ Ritonavir or Lopinavir (structures shown at the bottom of the figure). The RNA (a, f) and the products of the exonuclease reactions (b-e, g-j) were analyzed by MALDI-TOF MS. The signal intensity was normalized to the highest peak. The peak at $8165 \mathrm{Da}$ corresponds to the intact RNA (8157 Da expected). In the absence of the NS5A inhibitors, exonuclease activity caused nucleotide cleavage from the 3'-end of the RNA as shown by the 7 lower molecular weight fragments corresponding to cleavage of 1-7 nucleotides $(\mathrm{b}, \mathrm{g})$. In the presence of Ritonavir and Lopinavir, exonuclease activity was inhibited as shown by the reduced intensities of fragmentation peaks and increased intensity of the intact RNA peak (c-e, h-j).

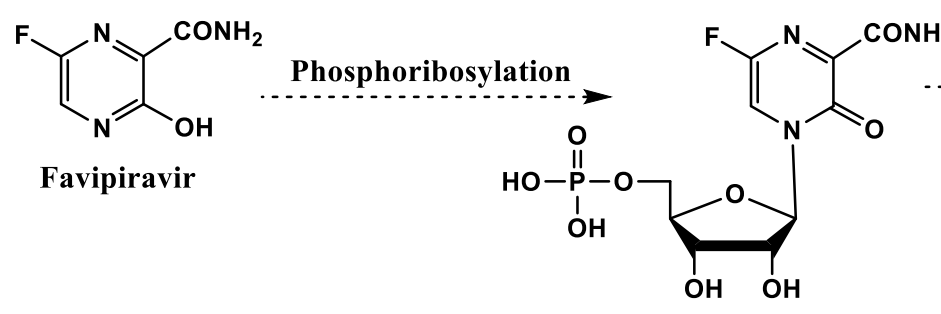

Favipiravir-RMP

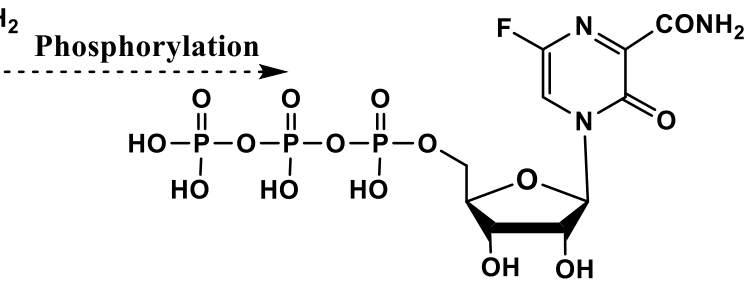

Favipiravir-RTP Active form

Fig. S-10 Mechanism of activation of Favipiravir. The nucleotide Favipiravir undergoes a phosphoribosylation reaction to form Favipiravir-ribofuranosyl-5'-monophosphate (RMP) followed by further phosphorylation to form the active triphosphate Favipiravir-ribofuranosyl-5'-triphosphate (RTP).

Table S-1 In vitro pharmacological parameters for representative viral RNA polymerase and HCV NS5A inhibitors against SARS-CoV-2 production in Calu-3 cells.

\begin{tabular}{|l|l|l|l|}
\hline & $\mathbf{E C}_{\mathbf{5 0}}$ & $\mathbf{C C}_{\mathbf{5 0}}$ & SI \\
\hline Drug & {$[\boldsymbol{\mu M}]$} & {$[\boldsymbol{\mu M}]$} & \\
\hline Remdesivir & $0.09 \pm 0.002$ & $480 \pm 55$ & 5333 \\
\hline Sofosbuvir & $6.2 \pm 0.3$ & $512 \pm 34$ & 82 \\
\hline Tenofovir (TDF) & $4.3 \pm 2.1$ & $91 \pm 25$ & 21 \\
\hline Favipiravir & $7.8 \pm 1.2$ & $670 \pm 118$ & 85 \\
\hline Pibrentasvir & $0.7 \pm 0.2$ & $22 \pm 3$ & 31 \\
\hline Ombitasvir & $0.4 \pm 0.05$ & $70 \pm 12$ & 175 \\
\hline Daclatasvir & $0.7 \pm 0.08$ & $38 \pm 5$ & 54 \\
\hline
\end{tabular}

$$
\text { SI }- \text { Selectivity index }=\mathrm{CC}_{50} / \mathrm{EC}_{50}
$$

